TUM-HEP-293/97

LBNL-41081

\title{
Dynamical Electro-Weak Symmetry Breaking with a Standard Model Limit ${ }^{\dagger}$
}

\author{
Manfred Lindner* and Erhard Schnapka** ${ }^{* *}$ \\ * Physik Department, Technische Universität München \\ James-Franck Str., D-85748 Garching b. München, Germany \\ Email: lindner@physik.tu-muenchen.de \\ ** Theoretical Physics Group \\ Ernest Orlando Lawrence Berkeley National Laboratory \\ University of California, Berkeley, California 94720, USA \\ Email: schnapka@lbl.gov
}

\begin{abstract}
We argue that a Standard Model decoupling limit is generically the necessary ingredient which makes scenarios of electro-weak symmetry breaking viable. This applies especially also to models of dynamical electro-weak symmetry breaking. Additional requirements are only that the mass predictions of a given model (e.g. predictions or theoretical limits on the Higgs or top mass) are consistent with existing data. We discuss the necessary ingredients for dynamical symmetry breaking and present a dynamically broken left-right-symmetric model as an example. The model exhibits such a decoupling limit, is phenomenologically viable and leads to interesting mass predictions and relations which are further examined.
\end{abstract}

\footnotetext{
${ }^{\dagger}$ To appear in "Heavy Flavours II", eds. A.J. Buras and M. Lindner, Advanced Series on Directions in High Energy Physics, World Scientific Publishing Co., Singapore

${ }^{\ddagger}$ Supported by the BASF AG and the Studienstiftung des deutschen Volkes.
} 


\section{Introduction}

In recent years the Standard Model has been able to describe the experimental data with impressive accuracy [1], including various different reactions and precision tests of radiative corrections. Despite the successes of the Standard Model, the current experiments mainly test the gauge structure. For the Higgs sector we know essentially only that the gauge symmetry is broken by some suitable vacuum expectation value. In the Standard Model the elementary Higgs sector leads to the famous hierarchy problem [2], due to the quadratic divergences associated with fundamental scalar particles. A solution to this problem probably involves the embedding of the Standard Model in new physics at the TeV-scale, and there are two main approaches: Either the quadratic corrections are cancelled due to restored supersymmetry (SUSY) above the SUSY breaking scale $\Delta \simeq T e V$, or some strongly interacting dynamical electro-weak symmetry breaking scenario provides form-factors (i.e. un-binding) and eliminates elementary Higgs bosons in the underlying theory.

Both routes have attractive features, but what can we learn from the current experimental situation? Within the framework of SUSY-GUTs one can obtain a remarkably accurate prediction of the weak mixing angle from gauge coupling unification. However, although being a nice feature, this is hardly direct evidence for supersymmetry. Apart from that, the so-called Minimal Supersymmetric Standard Model (MSSM) is unchallenged by current experimental data. This is however largely because in the MSSM all non-Standard Model effects can decouple if the soft supersymmetry breaking mass parameters are raised in the TeV-region and flavour changing effects are tuned to be small. In other words, the MSSM can hide sufficiently behind the Standard Model, at least as long as the experimental situation allows the Higgs mass to be below the MSSM-upper bound.

The situation may appear different for dynamical electro-weak symmetry breaking with a strongly interacting sector in the TeV-region. In naive Technicolor models, based on a rescaled version of QCD, one finds severe constraints from the existing data, and most of the original models are now ruled out by experiment. Note, however, that this is not a generic problem of dynamical symmetry breaking, but essentially the failure of any model where the effective Lagrangian does not have a Standard Model-limit. Both perturbative or non-perturbative scenarios of physics beyond the Standard Model with a decoupling limit are, on the other hand, phenomenologically acceptable, unless Higgs or other mass predictions disagree with experimental constraints. The question is thus if models of dynamical symmetry breaking with a Standard Model limit can be built.

There are genuine technical differences between low energy supersymmetry and models of dynamical electro-weak symmetry breaking which should not be used as an argument for or against either direction. While the MSSM is mostly perturbative and, therefore, its phenomenology relatively easy to analyse, dynamical symmetry breaking generically arises non-perturbatively. Therefore many quantities in such models are harder to calculate and predictions often very rough 1 . There is also no or little guidance from a greater picture

\footnotetext{
${ }^{1}$ For example, in a recently proposed Technicolor-like model [3] based on an approximate infrared
} 
compared to a supersymmetric framework.

Important are the conceptual differences between the approaches and ultimately, of course, future experiments will have to decide which scenario is realized in nature. Low energy supersymmetry may radiatively induce electro-weak symmetry breaking and leads to a rich phenomenology which can be studied perturbatively. At the same time, the hierarchy problem can be solved, provided that one finds a compelling (dynamical) model of supersymmetry breaking that leads to the desired soft-breaking terms in the TeV-region. As an alternative to models of supergravity-mediated SUSY-breaking [四], a variety of models have been proposed where SUSY breaking is mediated at low energies by standard gauge interactions [5]. Building such models has been helped by a significant improvement in understanding the non-perturbative dynamics of $\mathrm{N}=1$ supersymmetric gauge theories [6]. On the other hand, "immediate" dynamical electro-weak symmetry breaking is attractive from a simplistic point of view. Dynamical symmetry breaking is a natural effect in strongly interacting theories when an asymmetric ground state lowers the total energy of the system. Well known examples in other fields of physics are ferro magnetism and superconductivity, where in the latter case one finds a dynamical Higgs mechanism. Unfortunately, far less is know about the non-perturbative behaviour of non-supersymmetric field theories, compared to the supersymmetric case. Nevertheless it is worth to consider scenarios where the electro-weak gauge symmetry is dynamically broken by strong dynamics in the TeVregion. There might even be a sequence of layers of new physics, which perhaps also involves supersymmetry at scales well beyond the electro-weak scale.

In this article we would like to exemplify, that the current experimental situation essentially favours any framework which can "hide" behind the Standard Model. We will argue that it is possible to systematically build models of dynamical symmetry breaking with such a decoupling limit, which by construction may be consistent with the stringent constraints from the data. As an example, such an intrinsic decoupling limit can be found in models of top-condensation, where the Standard Model Higgs boson is replaced by a scalar $\bar{t} t$-bound-state, acting as the unitarity partner for the Goldstone bosons. Later in this article we will present a phenomenologically viable dynamically broken left-right-symmetric model based on this approach.

\section{The Standard Model Limit}

By now there exists direct evidence for all fermions and gauge bosons of the Standard Model and we know that the electro-weak gauge group is broken. There are experimental lower limits and theoretical (indirect and model dependent) upper limits for the Higgs mass, but there is no direct evidence for the existence of a Higgs particle. Existing data provides, however, additionally numerous restrictions for modifications of the Higgs sector. Examples

fixed point and near criticality, the additional effects of ordinary QCD can lead to electro-weak symmetry breaking. However, lattice simulations or better analytical understanding of non-perturbative effects will be needed to confirm the phenomenological viability of such a framework. 
are rare decays, FCNC effects, contributions to $R_{b}$ and other indirect effects via radiative corrections. Among those the so-called oblique corrections, commonly parametrized by the S-, T- and U- parameters, are particularly important. Thus, while a model of new physics attempting to explain electro-weak symmetry breaking need not feature an elementary Higgs boson, it certainly must be in accordance with all the above mentioned indirect constraints which are consistent with the Standard Model.

We think of a model of new physics as having a "Standard Model limit", if the parameters of the model can be chosen such that (within current experimental errors) the model becomes in a certain limit indistinguishable from the Standard Model, e.g., all effects from additional particles and interactions can be decoupled. If this is possible, it will allow a certain range of those parameters which are so far only poorly determined within the Standard Model, such as the mass of the Standard Model Higgs boson, certain mixing angles, details of CP violation or neutrino masses. Thus a Standard Model limit - if it exists - does not necessarily lead to every possible choice of Standard Model parameters, and examples for 'relic' mass restrictions (e.g., for the Higgs mass) will be seen below.

The minimal supersymmetric extension of the Standard Model (MSSM) exhibits such a Standard Model limit. To be phenomenologically viable, one must ensure sufficient separate conservation of the three lepton numbers, the suppression of flavour changing neutral currents (FCNC) as well as the smallness of the neutron and electron electric dipole moments. As emphasized in a recent review [7], this confines the MSSM to special, "exceptional" points in parameter space, and there are several attempts to explain this situation f. Once within this parameter regime, the virtual effects of supersymmetric particles can be decoupled from electro-weak observables at or below the $Z$-mass [8], when the masses of all supersymmetric particles are raised above $\sim 200 \mathrm{GeV}$. Especially, all the non-minimal Higgs bosons of the MSSM $\left(H^{0}, H^{ \pm}\right.$and $\left.A^{0}\right)$ can be decoupled, when the mass of the CP-odd Higgs boson is raised $\left(m_{A^{0}} \gg m_{Z^{0}}\right)$. In this limit the CP-even Higgs boson $h^{0}$ remains light, with Standard Model equivalent couplings to all Standard Model particles. This is because supersymmetry relates the quartic Higgs coupling (and mass) to gauge couplings, and after taking radiative corrections into account the CP-even Higgs bosons is always lighter than $\sim 125 \mathrm{GeV}$ [9]. Thus, due to this Standard Model limit, the MSSM can be consistent with electro-weak precision data, provided the additional "relic" mass restriction is met. I.e., the global fit for the Standard Model Higgs mass must be consistent with the upper bound on $h^{0}, m_{h^{0}} \lesssim 125 \mathrm{GeV}$.

Let us now see how the Standard Model limit can be realized in different scenarios with strongly interacting physics in the TeV-region with dynamical electro-weak symmetry breaking. Besides the issue of flavour changing neutral currents, it is very important to consider the so-called "oblique radiative corrections", which can be parametrized in the

\footnotetext{
${ }^{2}$ In the MSSM with minimal particle content and R-parity conservation assumed, the number of parameters of the model equals 124 [7]. One possibility to largely reduce this set of parameters, and to naturally solve the SUSY-flavour problem in the process, opens up in the context of gauge mediated supersymmetry breaking. The flavour blindness of gauge interactions leads to flavour blind soft breaking squark and slepton mass terms, which ensure the desired FCNC suppression.
} 
precision variables $S, T$, and $U$. These corrections are defined from the vacuum polarization amplitudes of $\gamma, W$, and $Z$ and the $\gamma Z$ mixing which have the form

$$
\begin{aligned}
\Pi_{\gamma \gamma} & =e^{2} \Pi_{Q Q}, \\
\Pi_{W W} & =\frac{e^{2}}{s^{2}} \Pi_{11}, \\
\Pi_{Z Z} & =\frac{e^{2}}{c^{2} s^{2}}\left(\Pi_{33}-2 s^{2} \Pi_{3 Q}+s^{4} \Pi_{Q Q}\right), \\
\Pi_{Z \gamma} & =\frac{e^{2}}{c s}\left(\Pi_{3 Q}-s^{2} \Pi_{Q Q}\right) .
\end{aligned}
$$

Here the relation $J_{Z}=J_{3}-s^{2} J_{Q}$ has been used. $J_{Q}$ is the electro-magnetic current, $s^{2}=\sin ^{2} \theta_{w}, c^{2}=\cos ^{2} \theta_{w}$, and the weak coupling constants have been expressed in terms of $e, s^{2}$ and $c^{2}$. The indices $i, j$ of $\Pi_{i j}$ on the right hand side indicate the relevant currents. The Standard Model contributions to the vacuum polarizations can be separated such that the remaining contributions of new physics are then functions of $q^{2} / M^{2}$, where $M^{2}$ is the mass of new, heavy particles. If $M^{2}$ is large enough one can expand in powers of $q^{2}$. Using QED Ward-Identities for $q^{2}=0$ the expansion leads at order $q^{2}$ to six coefficients:

$$
\begin{array}{rrr}
\Pi_{Q Q} & = & q^{2} \Pi_{Q Q}^{\prime}(0)+\ldots . \\
\Pi_{11} & = & \Pi_{11}(0)+q^{2} \Pi_{11}^{\prime}(0)+\ldots . \\
\Pi_{3 Q} & = & q^{2} \Pi_{3 Q}^{\prime}(0)+\ldots \\
\Pi_{33} & = & \Pi_{33}(0)+q^{2} \Pi_{33}^{\prime}(0)+\ldots
\end{array}
$$

When the three most precise measured observables, $\alpha_{e m}, G_{F}$ and $m_{Z}$, are used as input there remain three independent variables which parametrize effects of new physics. These three variables can be defined as 10

$$
\begin{aligned}
S & =16 \pi\left[\Pi_{33}^{\prime}(0)-\Pi_{3 Q}^{\prime}(0)\right], \\
T & =\frac{4 \pi}{s^{2} c^{2} m_{Z}^{2}}\left[\Pi_{11}(0)-\Pi_{33}(0)\right], \\
U & =16 \pi\left[\Pi_{33}^{\prime}(0)-\Pi_{11}^{\prime}(0)\right] .
\end{aligned}
$$

The last variable, $U$, is so small that it is currently irrelevant and will not be considered further. The variable $T$, measuring custodial SU(2) violating effects, and $S$, which is related to axial $\mathrm{SU}(2)$ and which is sensitive to the unitarity partner of Goldstone bosons, will both be discussed in more detail below.

It is instructive to consider the effects of naive Technicolor scenarios on oblique radiative corrections. The original models of this type, as well as more sophisticated versions like extended Technicolor (ETC) or "walking" Technicolor, have largely been ruled out in the 
past [11], and in the following we describe the basic difficulties. In the simplest version of these models of dynamical electro-weak symmetry breaking, the Goldstone boson decay constant of QCD is scaled up such that the correct $W$ mass arises. This leads (up to $N_{T C} \neq 3=N_{c}$ corrections) to a more or less fixed spectrum of QCD-like bound states in the TeV-region. Consequently the Techni-pions, the Goldstone bosons giving mass to $W$ and $Z$ in a dynamical Higgs mechanism, do not have a Higgs-like, i.e., scalar, unitarity partner 5 . Instead, the role of unitarity partners is played predominantly by low lying composite vector resonances, the Techni-rhos. Due to the QCD-like dynamics it is therefore not possible to obtain the Standard Model spectrum, where a suitable composite Higgs-like scalar mimics the Standard Model Higgs boson, and the remaining spectrum is decoupled. However, the main phenomenological problem of naive Technicolor is not per se the absence of the scalar partner of the Goldstone bosons, but the low mass of the Techni-rho resonances. We will see in a moment that low lying (composite or fundamental) vector-like states are in general a problem for the experimentally small precision variable $S$, even if there is a (composite or fundamental) Higgs particle. If a Higgs-like scalar is absent, like in Technicolor, then there exist of course upper bounds for the masses of vector states due to the unitarity of Goldstone boson scattering amplitudes. Such upper bounds for the masses of vector states do not exist in models where a Higgs particle exists in addition to extra vector states. An example is given by models which contain a $Z^{\prime}$, which can in principle become arbitrarily heavy.

The phenomenological problem with light vector states is that they can mix with the $W$ and $Z$-bosons, which is severely constrained by precision electro-weak data, i.e. the smallness of the $S$ parameter. This explains why in Technicolor the problem becomes even more severe as the number of Techni-colors $N_{T C}$ is increased, since for large $N_{T C}$ the ratio of Techni-rho mass and Goldstone boson decay constant becomes smaller, and the mixing with $W$ and $Z$ is increased. This effect can be seen best by expressing $S$ with the help of dispersion relations [10] as

$$
S=\frac{1}{3 \pi} \int_{0}^{\infty} \frac{d s}{s}\left[R_{V}(s)-R_{A}(s)-H(s)\right],
$$

where $R_{V}$ and $R_{A}$ measure the contribution of vector and axial-vector states, respectively. $R_{V}$ and $R_{A}$ are defined as the ratios of the cross sections of a photon which couples to the isospin current $J^{\mu 3}$ divided by the Compton process, in analogy to the famous $R$ ratio in QCD. The definition of $S$ depends on a reference Standard Model Higgs mass value which was often chosen to be $300 \mathrm{GeV}$. The function $H(s)$ allows one to remove the Standard Model Higgs contribution, e.g., in Technicolor by sending the Higgs mass into the continuum of bound states (i.e., $m_{H} \simeq 1 \mathrm{TeV}$ ). The function $H$ can be written as

$$
H(s)=\frac{1}{4}\left(1-\frac{m_{H}^{2}}{s}\right)^{3} \theta\left(s-m_{H}^{2}\right)
$$

which leads essentially to a small logarithmic dependence on the Higgs mass value.

\footnotetext{
${ }^{3}$ The so-called sigma particle would - if it exists - be broad and too heavy.
} 
In Technicolor the quantities $R_{V}$ and $R_{A}$ measure the sum of charges involved in $I=1$ vector resonances. One can estimate $S$ by parametrizing (similar to QCD) the resonance and the continuum contributions to $R_{V}$ and $R_{A}$ by delta and theta functions, respectively. The leading contributions come from the vector meson resonances (vector meson dominance), which leads to

$$
R_{V}(s)=12 \pi^{2} F_{\rho T}^{2} \delta\left(s-m_{\rho T}^{2}\right), \quad R_{A}(s)=12 \pi^{2} F_{a_{1} T}^{2} \delta\left(s-m_{a_{1} T}^{2}\right),
$$

where $m_{\rho T}$ and $m_{a_{1} T}$ are the Techni-rho and Techni- $a_{1}$ masses. With the help of the Weinberg sum rules [12] it is possible to express $F_{\rho T}^{2}$ and $F_{a_{1} T}^{2}$ as

$$
F_{\rho T}^{2}=\frac{m_{a_{1} T}^{2} F_{\pi}^{2}}{m_{a_{1} T}^{2}-m_{\rho T}^{2}}, \quad F_{a_{1} T}^{2}=\frac{m_{\rho T}^{2} F_{\pi}^{2}}{m_{a_{1} T}^{2}-m_{\rho T}^{2}},
$$

where $F_{\pi}=250 \mathrm{GeV}$. From this one obtains from $R_{V}$ and $R_{A}$ the following contribution to $S$ :

$$
S=4 \pi\left(1+\frac{m_{\rho T}^{2}}{m_{a_{1} T}^{2}}\right) \frac{F_{\pi}^{2}}{m_{\rho T}^{2}} .
$$

Using large $N_{c}$ rescaling relations between Technicolor and QCD one finds [13]

$$
\frac{m_{\rho T}^{2}}{m_{a_{1} T}^{2}}=\frac{m_{\rho}^{2}}{m_{a_{1}}^{2}} \quad \frac{F_{\pi}^{2}}{m_{\rho T}^{2}}=N_{D} \frac{N_{T C}}{3} \frac{f_{\pi}^{2}}{m_{\rho}^{2}} .
$$

With $f_{\pi}=93 \mathrm{MeV}, m_{\rho}=770 \mathrm{MeV}$ and $m_{a_{1}}=1260 \mathrm{MeV}$ this results in $S \simeq 0.25 N_{D} N_{T C} / 3$, where $N_{T C}$ is the number of Techni-colors and $N_{D}$ is the number of weak doublets. The continuum contributions to $R_{V}$ and $R_{A}$ can be parametrized by theta-functions and lead to logarithmic mass dependencies, while the resonances lead to $1 / m_{\rho T}^{2}$ contributions. These $1 / m_{\rho T}^{2}$ contributions are usually dominant unless logarithmic contributions proportional to $N_{T C}^{2}$ become for $N_{T C}>8$ equally or even more important.

If one evaluates $R_{V}$ and $R_{A}$ using a more detailed analysis of QCD data and large $N_{c}$ rescaling one finds 10

$$
S=0.3 N_{D} \frac{N_{T C}}{3}
$$

which must be confronted with the value of $S$ extracted from global fits to existing data. For such fits it is necessary to specify the top quark mass and the Higgs mass of the Standard Model, and typically the values $m_{t}=175 \mathrm{GeV}$ and $m_{H}=300 \mathrm{GeV}$ are assumed [14]. For comparison with Technicolor models the reference Higgs mass is sometimes shifted to $1 \mathrm{TeV}$, which leads to

$$
S=-0.26 \pm 0.16
$$

The negative sign expresses the fact that the preferred Higgs mass of the Standard Model is much smaller than $1 \mathrm{TeV}$, and today the best fit is obtained [15] for $m_{H} \approx 121 \mathrm{GeV}$. Comparing equation (19) with the prediction of naive Technicolor, equation (18), one finds 
the smallest disagreement at the $3 \sigma$ level for $N_{D}=1$ and $N_{T C}=3$. More Technicolor doublets and/or a larger Technicolor group lead to even larger deviations from the data.

The above expression for $S$, equation (12), applies in principle for any theory with composite or fundamental vectors and/or axial-vector states which couple to $J^{\mu 3}$. For example, one could study $Z^{\prime}$ models in this way and find a $1 / m_{Z^{\prime}}^{2}$ dependence from the mixing of the $Z^{\prime}$ with $Z$. This leads to unacceptable contributions to $S$ if the extra vector state becomes light. However, these contributions are not as severe as in Technicolor since the $Z^{\prime}$ couplings are usually chosen to be perturbative (i.e. small) and the color factor $N_{T C}$ is absent.

Another severe problem of many Technicolor scenarios stems from additional extra Techni-fermion doublets. The problem shows up in the precision variable $T$ defined above and emerges in a more general context for new fermionic doublets, i.e., it is not only a genuine Technicolor problem. Since extra fermions are not observed such a $\mathrm{SU}(2)_{L}$ doublet is required to be massive with masses well above one hundred GeV. When the massive propagators for extra doublets of fermions are written as

$$
S_{j}=\frac{i}{\not p-\Sigma_{j}\left(p^{2}\right)},
$$

where $j=U, D$ for a new doublet and $j=t, b$ for the top and bottom quark, then the contribution to $T$ is given by [16]

$$
T=\frac{-N_{c}}{16 \pi^{2} \alpha_{e m} v^{2}} \int_{0}^{\infty} d k^{2} \frac{k^{4}\left(\Sigma_{U}^{2}-\Sigma_{D}^{2}\right)^{2}}{\left(k^{2}-\Sigma_{U}^{2}\right)^{2}\left(k^{2}-\Sigma_{D}^{2}\right)^{2}} .
$$

Here $N_{c}$ is the number of colors or Techni-colors and $v \simeq 246 \mathrm{GeV}$. If we insert $\Sigma_{t}=m_{t}$ and $\Sigma_{b}=0$ into equation (21) one finds the well known, leading Standard Model top mass contribution to the $T$-parameter,

$$
T_{S M}=\frac{N_{c}}{16 \pi^{2} \alpha_{e m}} \frac{m_{t}^{2}}{v^{2}}=\frac{N_{c}}{16 \pi \sin ^{2} \theta_{W} \cos ^{2} \theta_{W}} \frac{m_{t}^{2}}{M_{Z}^{2}}
$$

Equation (21) is also valid for new heavy doublets. In Technicolor, for example, ordinary quark and lepton masses (like the top mass) must be generated by so-called Extended Technicolor [17] interactions between quarks and Techni-quarks. The coupled system of gap equations leads, in a first approximation [18], to the relation

$$
\Sigma_{U}-\Sigma_{D}=\Sigma_{t}
$$

where $\Sigma_{U}$ and $\Sigma_{D}$ are the mass functions of the Technicolor doublet. Assuming that equation (23) is valid and approximating $\Sigma_{i}$ as $\Sigma_{i}=m_{i} \Theta\left(\Lambda^{2}-p^{2}\right)$, equation (21) yields corrections to the Standard Model value of $T$ :

$$
\begin{aligned}
T_{\text {total }} & =T_{S M}+T \\
& =\frac{N_{c} m_{t}^{2}}{16 \pi^{2} \alpha_{e m} v^{2}}\left(1+\frac{4}{9} N_{T C}+\frac{m_{t}^{2}}{\Lambda^{2}}+\frac{4}{3} N_{T C} \frac{m_{U}^{2}}{\Lambda^{2}}+\mathcal{O}\left(m^{4} / \Lambda^{4}\right)\right)
\end{aligned}
$$


For $\Lambda \rightarrow \infty$ this expression becomes the result usually quoted in the literature [18]. For finite $\Lambda$ we can read off the $m_{t}^{2} / \Lambda^{2}$ correction to equation (25), and in addition a term proportional to $m_{U}^{2} / \Lambda^{2}$. For sufficiently large $\Lambda$ these $1 / \Lambda^{2}$ terms are small and can be omitted. In this limit the value of $T$ is given by $4 / 9 \cdot N_{T C} \cdot T_{S M}$, which is excluded by phenomenology due to the excellent agreement of the experimental value of $T$ with its Standard Model value. One might argue that equation (23) is very model dependent and may be generalized by a more complex relation, but it is not easy to obtain the top-bottom mass splitting without sizable corrections to $T$.

As mentioned earlier, the problems of extra doublets in Technicolor have a more general scope. Any theory with extra doublets and a similar mechanism to explain the top-bottom mass difference faces similar problems. If the mechanism which explains the top-bottom mass splitting induces a $U-D$ splitting proportional to $K \cdot m_{t}$, where $K$ is typically expected to be of order unity, then this will lead to $T=4 / 9 \cdot K^{2} N_{c} T_{S M}$. Unless $K$ is very tiny, this leads again to amounts of custodial $\mathrm{SU}(2)$ violation (measured by $T$ ) too large to be reconciled with precision measurements.

This discussion shows that two ingredients are disfavoured when constructing models of dynamical symmetry breaking with a Standard Model limit: Low lying vector states lead to undesired contributions to the S-parameter, and additional fermionic SU(2)-doublets cause excessive violation of custodial SU(2) symmetry, unless they are degenerate in mass.

At the end of this section we would like to emphasize, that neither the Standard Model limit properties of the MSSM nor the problems with naive Technicolor stem generically from a supersymmetric or dynamical mechanism of electro-weak symmetry breaking. The phenomenological viability or failure is to a large extent simply the presence or absence of the mentioned Standard Model limit, due to the lack of deviations of the experimental precision data from the Standard Model. In other words, this can be seen as the success of models which have a limit, where the low energy Lagrangian becomes effectively the Standard Model with a single Standard Model-like Higgs boson. In this respect low lying vector particles and extra fermionic doublets, as they appear in naive Technicolor-models, are disfavoured.

Note that it is irrelevant for this discussion whether the resonances (vector or scalar) are elementary or composite. Therefore it appears promising to develop models of dynamical symmetry breaking with purely scalar unitarity partners for the Goldstone bosons. With an effective or composite Higgs state, instead of Techni-rho like vector resonances, such models can include a Standard Model limit in the sense described earlier, and thus generically have a better chance to be phenomenologically viable. Future precision measurements may ultimately confirm specific deviations between the Standard Model and the data (e.g. in quantities like $R_{b}$, etc.). These deviations might then be explained more easily by departing from the Standard Model limit, i.e., by lowering some of the additional states, which are expected close to the continuum. Alternatively, of course, they might rule out a particular model. In any case, the underlying dynamics of such a scenario will likely lead to restrictions in the mass of whatever assumes the role of the Higgs boson in the Standard Model. In the 
MSSM this is the CP-even scalar $h^{0}$ which mass is bounded from above $(\leq 125 \mathrm{GeV})$ by the underlying dynamics, in a dynamical scenario considered here it will be a composite Higgs boson.

\section{Guidelines for Model Building}

The attempt to construct a viable model of dynamical electro-weak symmetry breaking may be viewed as a bottom-up approach, guided by the current phenomenological situation. As we saw in the previous section, the data so far favours any model with a Standard Model limit, and we will identify the ingredients of symmetry breaking that naturally lead to such a limit. In this context it is important to carefully distinguish between the Higgs mechanism (i.e. the Goldstone bosons "eaten" by gauge bosons, serving as their longitudinal degrees of freedom) and a physical Higgs particle. While the scalar Goldstone bosons are simply a consequence of broken global symmetries, irrespective of the nature of symmetry breaking (perturbatively or tree level), the existence or non-existence of a Higgs particle is a feature of the particular field theory considered.

The Higgs mechanism for a specific gauge symmetry breaking pattern requires only an operator $\hat{O}$ with the following properties:

- $\langle\hat{O}\rangle \neq 0$, i.e. a "condensate"

- Lorentz invariance of the vacuum requires that $\hat{O}$ must be scalar

- $\hat{O}$ must transform non-trivially under the gauge group to be broken, and as a singlet under the desired unbroken subgroups.

Note that $\hat{O}$ does not have to be a fundamental scalar Higgs field. For the case of the Standard Model gauge group $\mathrm{SU}(3)_{c} \times \mathrm{SU}(2)_{L} \times \mathrm{U}(1)_{Y}$ the operator $\hat{O}$ should be a doublet of $\mathrm{SU}(2)_{L}$ with $Y=1$ for the hypercharge 1 . The condensate $\langle\hat{O}\rangle \neq 0$ then breaks the global SU $(2)_{L} \times \mathrm{U}(1)_{Y}$ symmetry, which implies the existence of Goldstone bosons which can be eaten by W's and Z's. In unitary gauge the operator can be expanded as

$$
\hat{O}=(\langle\hat{O}\rangle+\delta \hat{O}) e^{i \varphi_{a} T_{a}}
$$

and if a $|D \hat{O}|^{2}$ term is present in the effective Lagrangian with $\langle\hat{O}\rangle \neq 0$, the Goldstone bosons $\varphi_{a}$ are absorbed by the corresponding gauge bosons. The Higgs mechanism requires only the existence of a suitable condensate $\langle\hat{O}\rangle \neq 0$ and operates on the basis of symmetries, it does not depend on either the fundamental or non-fundamental nature of $\hat{O}$ and/or the presence of $\delta \hat{O}$.

\footnotetext{
${ }^{4} \hat{O}$ could in principle have different quantum numbers, but phenomenological evidence like the smallness of $\Delta \rho$ lead to strong constraints.
} 
The interactions of the Goldstone bosons can thus be understood in terms of the involved symmetries and the corresponding Ward-Identities, and the details of the interaction responsible for symmetry breaking become almost irrelevant. This is well established in QCD, where even a Nambu-Jona-Lasinio description of chiral symmetry breaking (which has almost nothing to do with QCD, but can be arranged to break the chiral symmetries correctly) leads to a remarkable good description of pion interactions.

On the other hand, $\delta \hat{O}$ or other non-scalar excitations of the vacuum are not related to the symmetries of the theory and may or may not include a fundamental or composite physical scalar Higgs particle $H$. If $\hat{O}$ is fundamental and $\delta \hat{O}$ is omitted completely, one arrives at the non-linear sigma model. This is fine with respect to symmetry breaking, but renormalizability is lost, which is an essential feature of a fundamental scalar theory. Unless new physics is very close, Goldstone boson scattering amplitudes are unbounded and unitarity is violated. One way out is to postulate a fundamental scalar Higgs field $H$ which can be grouped together with the Goldstone bosons in a $\mathrm{SU}(2)_{L}$ doublet $\Phi$, and where $H$ can act as "unitarity partner" for the Goldstone bosons. Unless new physics (i.e. some extra scale) is close renormalizability requires in addition that the Higgs potential $V(H)$ has only a mass term and $\lambda \Phi^{4}$ interactions.

Note what happens if $\delta \hat{O}$ corresponds to a composite operator. In this case the Goldstone bosons are composite as well, and the Higgs mechanism will work as before. However, the remaining spectrum of the theory will typically be rich with an effective interaction La-

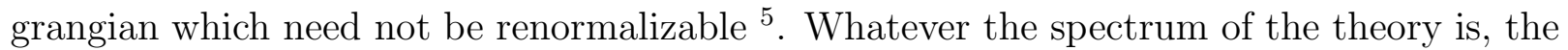
effective Lagrangian must contain some resonances which act as unitarity partner for the Goldstone bosons. The simplest scenarios would be either a composite Higgs like state (i.e. an effective Standard Model) or suitable vector resonances with the remaining spectrum located close to the continuum, i.e., at some high scale $\Lambda$. The details of the spectrum remain a dynamical issue, depending on the interactions of the underlying model. In QCD-like theories, for example, rho-like vector resonances emerge in the effective Lagrangian while a physical Higgs particle is absent. One can, however, choose interactions which lead to a composite Higgs $H$ instead. Note that the existence of a composite scalar $H$ allows a richer interaction potential and other resonances in addition. Thus a composite Higgs does not automatically lead to an effective Standard Model.

We can now discuss how the Standard Model limit can be reached systematically in dynamical symmetry breaking models:

- At first a suitable symmetry breaking pattern must be considered in order to arrive at the correct spectrum of Goldstone bosons. Care should be taken to avoid problems with so-called pseudo Goldstone bosons, since, being light, they lead easily to phenomenological problems.

- The existing data for the precision variable $S$ favours a spectrum where a Higgs-like

\footnotetext{
${ }^{5} \mathrm{An}$ example is again chiral symmetry breaking in QCD in the limit where pions are massless. Only the underlying theory - here QCD - should be based on fundamental fields and be renormalizable.
} 
scalar plays the role of the unitarity partner of the Goldstone bosons, as explained in section 2. Vector-like states with SU(2)-quantum numbers are disfavoured, and thus one is lead to consider scenarios which differ significantly from QCD.

- Custodial $\mathrm{SU}(2)$ violation measured by $T \simeq m_{t}^{2}$ agrees very well with the Standard Model value. This strongly disfavours scenarios which have sizable extra custodial $\mathrm{SU}(2)$ violating effects, such as additional fermionic doublets beyond the Standard Model. Unless special care is taken the mechanism responsible for the top-bottom mass splitting will lead to huge effects via extra doublets. Thus, in order to avoid fine-tunings or special choices, we avoid extra fermionic doublets beyond the Standard Model.

- The absence of flavour changing neutral currents (FCNC's) beyond the Standard Model easily becomes a problem in models where the heavy top mass is explained after the electro-weak symmetry is broken. A well known example is the generation of quark masses in extended Technicolor. This might suggest that the heavy top mass is intimately related to electro-weak symmetry breaking, which leads to the idea of top-condensates.

Dynamical symmetry breaking models along these guidelines should be phenomenologically much more viable than, e.g., naive Technicolor since the model has a Standard Model limit. The above choices are not very artificial and the physically most interesting point is probably to understand which sort of dynamics produces the scalar spectrum.

\section{The BHL Example}

There is a nice prototype model which implements the Standard Model limit in a minimal way: The so-called BHL model [19] of electro-weak symmetry breaking. The idea is here to eliminate the fundamental Higgs field in the Standard Model and to introduce instead a new attractive interaction, which leads to the formation of a $t \bar{t}$-condensate. In terms of the discussion given above, the elementary Higgs field of the Standard Model gets replaced by the bi-fermion composite operator

$$
\hat{O} \simeq \bar{Q}_{L} t_{R}
$$

with exactly the Standard Model-Higgs quantum numbers. We found previously that scalars are favoured as unitarity partners of the Goldstone bosons due the constraints on the $\mathrm{S}$ parameter. In addition, in this approach the generation of the large top mass occurs within the process of gauge symmetry breaking. Therefore $m_{t}$ is naturally of the order of the electro-weak scale and need not be artificially generated at the cost of possibly large FCNC's.

The interaction responsible for triggering the condensation is assumed to have its origin in new yet unspecified physics above some high-energy scale $\Lambda$. At lower energies this sector 
of new physics manifests itself through non-renormalizable interactions between the usual fermions and gauge bosons, where for energies $E \ll \Lambda$ the lowest dimensional four-fermion operators are most important. Thus, at the scale $\Lambda$ the Lagrangian can be given by the gauge-kinetic terms for the known chiral fermions and gauge bosons, plus a gauge invariant four-fermion interaction term

$$
\mathcal{L}=\mathcal{L}_{k i n}(g, f)+\mathcal{L}_{4 f}^{B H L}
$$

The gauge invariant four-fermion operator which is needed for a condensation of the operator $\hat{O}$ [see (26)] in the $t \bar{t}$-channel is given by [20, 21, 19, 22]

$$
\mathcal{L}_{4 f}^{B H L}=G\left(\bar{Q}_{L i} t_{R}\right)\left(\bar{t}_{R} Q_{L i}\right)
$$

Here $Q_{L}$ is the left-handed doublet of the third generation quarks, $G$ is a dimensionful coupling constant, $G \sim \Lambda^{-2}$, and it is implied that the color indices are summed over within each bracket.

The above Lagrangian represents a gauged NJL-model, where condensation and electroweak symmetry breaking can occur for $G>G_{\text {critical }}$. The model can be studied analytically in the large $N_{c}$ (number of colors) limit in the so-called NJL or fermion bubble approximation [6, [23, 24]. In auxiliary field formalism one can define the local composite operator $\varphi:=-G \bar{t}_{R} Q_{L}$, which allows the Lagrangian (27) to be rewritten with the help of the equations of motion into

$$
\mathcal{L}=\mathcal{L}_{k i n}(g, f)-\bar{Q}_{L} \varphi t_{R}-\bar{t}_{R} \varphi^{+} Q_{L}-G^{-1} \varphi^{+} \varphi
$$

Integrating out the degrees of freedom between $\mu<\Lambda$ and $\Lambda$ radiatively generates the additional renormalizable terms $\rceil$ for (29). The resulting effective Lagrangian at $\mu<<\Lambda$ can be written as

$$
\begin{aligned}
\mathcal{L}_{\mathrm{eff}}= & \mathcal{L}_{k i n}(g, f)+\delta \mathcal{L}_{k i n}(g, f) \\
& +Z_{\varphi}|D \varphi|^{2}-\left(1+\delta g_{t}\right)\left(\bar{Q}_{L} \varphi t_{R}+\bar{t}_{R} \varphi^{+} Q_{L}\right) \\
& -\left(G^{-1}-\delta M^{2}\right) \varphi^{+} \varphi-\frac{\delta \lambda}{2}\left(\varphi^{+} \varphi\right)^{2} .
\end{aligned}
$$

This is the effective Lagrangian for the composite operator $\hat{O} \equiv \varphi$, and the CJT effective potential [25] in the fermion bubble approximation can easily be read off:

$$
V_{\mathrm{eff}}(\varphi)=\left(G^{-1}-\delta M^{2}\right) \varphi^{+} \varphi+\frac{\delta \lambda}{2}\left(\varphi^{+} \varphi\right)^{2}
$$

The terms $\delta M^{2}$ and $\delta \lambda$ follow from the one loop diagrams with two and four external composite operators connected via four-fermion (effectively Yukawa) vertices with weight

\footnotetext{
${ }^{6}$ We use the well known abbreviation NJL though the paper of Vaks and Larkin was received and published first.

${ }^{7}$ For $\Lambda \simeq v$ many non-renormalizable terms would be generated as well.
} 
$G^{-1}$. The potential (31) leads to symmetry breaking for small $G^{-1}$, i.e. large enough $G>G_{\text {critical }}$. Up to the unconventionally normalized kinetic term, the $\varphi$-sector in the effective Lagrangian (30) looks like the Higgs sector of the Standard Model. The difference is that here the Higgs is composite, and the scale dependence on the infrared cutoff $\mu$ must be such that all the quantum effects generated in (30) disappear at $\mu=\Lambda$. These are the so-called "compositeness conditions"

$$
Z_{\varphi} \stackrel{\mu^{2} \rightarrow \Lambda^{2}}{\longrightarrow} 0 ; \quad \delta M^{2} \stackrel{\mu^{2} \rightarrow \Lambda^{2}}{\longrightarrow} 0 ; \quad \delta \lambda \stackrel{\mu^{2} \rightarrow \Lambda^{2}}{\longrightarrow} 0 .
$$

The rescaling $\varphi \longrightarrow \varphi / \sqrt{Z_{\varphi}}$ normalizes the kinetic term for the composite Higgs field to unity. Rewriting the compositeness conditions in this normalization one finds the boundary conditions which the Standard Model must fulfill if the Higgs particle emerges from top condensation.

These boundary conditions constitute restrictions on the parameter space of the low energy effective theory, and therefore lead to predictions which can be verified against experimental data. In the fermion bubble approximation the symmetry breaking and mass generation is calculable by minimizing the effective potential in equation (31). This procedure is equivalent to computing a self-consistent dynamical top mass by solving the gap equation and yields a prediction of $m_{t}$ in terms of the $W$-boson (see section 5 ), which depends logarithmically on the scale of new physics $\Lambda$. In addition one obtains (in bubble approximation) the relation $m_{H}=2 m_{t}$ for the Higgs boson mass, which is very suggestive for the Higgs being a $\bar{t} t$ bound-state. For $\Lambda \approx 10^{15} \mathrm{GeV}$ one finds a value of $m_{t} \approx 165 \mathrm{GeV}$, while $\Lambda \simeq \mathrm{TeV}$ leads to a top mass of a few hundred GeV. To obtain a phenomenologically acceptable value for the top quark mass one has to tune the four-fermion coupling constant $G$ extremely close to its critical value to allow $m_{t} / \Lambda$ to be tiny. It has been shown [19] that this is equivalent to the usual fine-tuning of the Higgs boson mass in the Standard Model. Thus, the gauge hierarchy problem is not solved in the top-condensate approach $\$$.

However, the bubble approximation employed so far is a rather crude approximation of the full dynamics and ignores important effects of the full theory, such as QCD corrections and the contributions of propagating composite scalars. An elegant way to incorporate these effects is to impose the above boundary conditions (32) on the renormalization group flow (RGE) of the full low energy theory, the Standard Model. The top mass prediction is then governed by the so-called infrared quasi fixed-points [27] of the top Yukawa coupling. Due to the focusing one obtains rather reliable predictions, mostly independent of nonperturbative effects close to the cutoff. It turns out that the improved top mass predictions are systematically higher than in bubble approximation. For a desirable low value for the cutoff in the TeV-range the top mass is too large by a factor of two. Even for undesirably large scales of $\Lambda>10^{15} \mathrm{GeV}$ one finds values around $m_{t}=220-240 \mathrm{GeV}$ [19], which are about 30 percent too high. Therefore, the BHL model is phenomenologically unacceptable due to an embedding relic: The top mass prediction.

\footnotetext{
${ }^{8}$ It has been claimed in [26] that taking into account the loops with composite Higgs scalars results in the automatic cancellation of quadratic divergences. Here we do not discuss this possibility further.
} 
Even though the BHL model is ruled out by the top mass prediction it demonstrates how the decoupling of physics beyond the Standard Model can be achieved in dynamical symmetry breaking models. At scales far below the scale of new physics $\Lambda$, the effective theory features a symmetry breaking sector with a scalar (composite) boson with exactly the quantum numbers of the Standard Model Higgs boson. From the low energy point of view, the model is indistinguishable from the Standard Model, i.e., a Standard Model limit, and the only remnant is the prediction for the top mass. It is therefore interesting to see which kind of theories at higher energies could justify the effective non-renormalizable four-fermion interactions of the BHL model with a cutoff. The four-fermion term of the model changes under Fierz transformation into the remarkable simple structure:

$$
G \bar{L} t_{R} \overline{t_{R}} L \stackrel{\text { Fierz }}{\longrightarrow}-\frac{G}{2}\left(\bar{L} \gamma_{\mu} L\right)\left(\bar{t}_{R} \gamma_{\mu} t_{R}\right)
$$

It is now easy to see that the four-fermion structure of the BHL model may be related to the exchange of suitable massive, strongly coupled vector bosons. Thus, such a scenario might be justified within a renormalizable theory with extended gauge group where the massive propagator has been integrated out. A number of renormalizable gauge theory models have been proposed along this line [28, 29, 30, 31]. Note that the dynamics of such scenarios deviates clearly from QCD in this picture. There are even hints for interesting confinement-Higgs dualities which might play a role in such models 29.

\section{More Condensates}

Most attempts to modify the BHL model have a common problem: They tend to produce a top mass which is unacceptably high, even for very high values for the scale of new physics. We will see that this is not an accident, but occurs systematically in scenarios of dynamical electro-weak symmetry breaking which is driven by a top condensate alone. If a top condensate breaks the electro-weak symmetry, then for an asymptotically free theory the dynamically generated top propagator can be written as

$$
S_{t}=\frac{i}{p-\Sigma_{t}\left(p^{2}\right)}
$$

where

$$
\Sigma\left(p^{2}\right) \stackrel{p^{2} \rightarrow \infty}{\longrightarrow} 0
$$

The dynamically generated self energy $\Sigma_{t}$ can be related to the Goldstone boson decay constant $F_{ \pm}$, which is induced by the condensate, by virtue of the so-called Pagels-Stokar relations [32]:

$$
F_{ \pm}^{2}=\frac{N_{c}}{32 \pi^{2}} \int d k^{2} \frac{\Sigma_{t}^{2}\left(k^{2}\right)}{\left(k^{2}-\Sigma_{t}^{2}\left(k^{2}\right)\right)} .
$$

These relations are derived primarily on the basis of Ward identities and are therefore valid beyond the approximations used to derive $\Sigma_{t}(p)$. Note that the integral in (36) is formally 
log divergent, but finite if the asymptotic behaviour of equation (35) is taken into account. Equation (36) is a very powerful relation between the dynamically generated top mass and the Goldstone boson decay constant, and also the $\mathrm{W}$ mass, since after the charged Goldstone boson is absorbed by $W$ one obtains $m_{W}^{2}=g_{2}^{2} F_{ \pm}^{2}$. It is instructive to observe that the integral on the right hand side of the Pagels-Stokar relation, equation (36), feels the structure of $\Sigma_{t}$ only on a logarithmic scale. Thus, for large values of $\Lambda$, the integral is dominated by contributions coming from scales both far away from $\Lambda$ and the electro-weak scale, i.e. from regions where $\Sigma$ should (up to logarithmic RGE running) essentially be flat. In this case it is a good approximation to express $\Sigma$ by its 'mean' height and extension:

$$
\Sigma_{t}\left(p^{2}\right)=m_{t} \Theta\left(\Lambda^{2}-p^{2}\right)
$$

To be more precise, this should be a valid approximation except for small values of $\Lambda$, but those values anyway lead to values of $m_{t}$ that are by far too large. Inserting equation (37) into equation (36) and solving for the top mass, one finds

$$
m_{t}^{2}=\frac{32 \pi^{2} m_{W}^{2}}{N_{c} g_{2}^{2} \ln \left(\Lambda^{2} / m_{t}^{2}\right)},
$$

which is exactly the relation obtained in the BHL model in bubble approximation. This makes sense, since the structure of the ansatz (37) corresponds to a Nambu-Jona-Lasinio gap equation. Corrections to this relation come, like in the BHL model, from other, weak gauge contributions and are expected to be moderate and model dependent. Thus, the Pagels-Stokar relation explains why many variants of the BHL model, like two Higgs doublets or the supersymmetric version, produce a similar top mass, a value too high to fit the data. On the other hand, inserting $m_{t}=175 \mathrm{GeV}$ in equation (38) one finds a $W$ mass which is too small for any $\Lambda$. Therefore, in order to obtain a viable relation between $\Lambda$ and the top mass, one is led to consider more complex symmetry breaking scenarios, with more condensates and/or more Yukawa couplings.

Given the success of renormalizable gauge theories, it appears attractive to relate extra condensates to more complex symmetry breaking patterns of extended gauge sectors. An appealing class of models where such ideas can be exemplified are dynamically broken left-right-symmetric theories. In such models, usually a condensate in the leptonic sector breaks left-right symmetry as a first step, and a second (or more) condensate(s) cause electro-weak symmetry breaking. We will show in the next section how dynamical symmetry breaking can be implemented in left-right-symmetric theories, along the guidelines presented in section 3, to give a model with a Standard Model limit. In particular, we will find a modified relation between $m_{t}$ and $\Lambda$ which is phenomenologically acceptable - even for low values of $\Lambda$. 


\section{A Left-Right-Symmetric Model with Decoupling Limit}

Left-right-symmetric models based on the gauge group $\mathrm{SU}(2)_{L} \times \mathrm{SU}(2)_{R} \times \mathrm{U}(1)_{B-L} \times \mathrm{SU}(3)_{c}$ have many attractive features [33, 34]. The U(1)-group corresponds to baryon minus lepton number and thus has a somewhat more intuitive interpretation than the weak hypercharge of the Standard Model. Furthermore, parity violation is explained as yet another step of spontaneous gauge symmetry breaking. All known quarks and leptons fit nicely in the fundamental representation of the gauge group, and the well known see-saw mechanism can be employed to obtain very light masses for the left-handed neutrinos.

The symmetry breaking sequence required by phenomenology proceeds in two steps, first the breaking of parity down to the Standard Model gauge group at an energy scale $\mu_{R}$, and second the usual electro-weak symmetry breaking:

$$
\begin{gathered}
\mathrm{SU}(2)_{L} \otimes \mathrm{SU}(2)_{R} \otimes \mathrm{U}(1)_{B-L} \\
\downarrow \mu_{R} \\
\mathrm{SU}(2)_{L} \otimes \mathrm{U}(1)_{Y} \\
\downarrow \mu_{\text {ew }} \\
\mathrm{U}(1)_{e m}
\end{gathered}
$$

In conventional left-right-symmetric models this breaking sequence is realized by introducing an elementary scalar Higgs sector. Naturally in this case the scalar sector is much larger than in the Standard Model, with of the order of twenty parameters to be adjusted to obtain the desired symmetry breaking pattern. In the light of the above considerations, it will be interesting to investigate whether the above symmetry breaking scenario can be realized in a dynamical model with composite Higgs bosons.

In the following we discuss composite model building in the framework of left-right symmetric theories, in analogy to the BHL model. We will show that the compositeness of scalars poses non-trivial constraints on the models that can be realized, and finally present a phenomenological viable left-right-symmetric model, where the correct vacuum structure and symmetry breaking sequence emerges dynamically [35, 36]. Essentially the symmetry breaking in this composite model involves at least two condensates, one large hybrid condensate in the neutrino sector to break parity, and, in the simplest case, a $t \bar{t}$ condensate to break the electro-weak symmetry. As it turns out, the general case gives three condensates, two of which will be in the quark sector and appear as the two vacuum expectation values of the bi-doublet composite scalar $\varphi$. These two condensates break the electro-weak gauge symmetry and, as we pointed out in the last section, provide the necessary degree of freedom to obtain viable top and bottom masses. 


\subsection{A First Attempt}

As in the BHL approach, we will only consider the usual fermions and gauge bosons of the model as elementary particles, with no fundamental Higgs scalars being present, and in addition introduce a set of relevant left-right-symmetric four-fermion interactions, representing yet unspecified new physics at a high energy scale $\Lambda$. Thus the Lagrangian will be of the form

$$
\mathcal{L}=\mathcal{L}_{\text {kinetic }}^{L R}+\mathcal{L}_{4 f}
$$

A useful framework to study models with composite Higgs bosons is the auxiliary field technique. The four-fermion interactions are rewritten in terms of Yukawa couplings of the fermions with newly introduced static auxiliary fields with appropriate quantum numbers. The non-propagating scalars come with a heavy mass term of the order of $G_{4 F}^{-1} \approx \Lambda^{2}$, but no kinetic term and no quartic interactions on tree level. Since the modified Lagrangian of the system is quadratic in these auxiliary fields they can always be integrated out in the functional integral [37]. Equivalently, one can use the equations of motion for these fields to express them in terms of the fermionic degrees of freedom. After substituting the resulting expressions into the auxiliary Lagrangian one reproduces the initial four-fermion structures. The static auxiliary fields can acquire gauge-invariant kinetic terms and quartic self-interactions through radiative corrections and become physical propagating scalar fields at low energies provided that the corresponding gap equations are satisfied [19]. The kinetic terms and mass corrections can be derived from the 2-point Green function, whereas the quartic couplings are given by the 4-point functions. Given the Yukawa couplings of the scalar fields one can readily calculate these functions in the fermion bubble approximation, in which they are given by the corresponding 1-fermion-loop diagrams.

Before presenting the final model, we will further consider the type of composite scalars we wish to obtain, and the constraints imposed by a correct symmetry breaking sequence. In the most popular left-right-symmetric model the Higgs sector consists of a bi-doublet $\varphi \sim$ $(2,2,0)$ and two triplets, $\Delta_{L} \sim(3,1,2)$ and $\Delta_{R} \sim(1,3,2)$, representations in parentheses corresponding to $\mathrm{SU}(2)_{L} \otimes \mathrm{SU}(2)_{R} \otimes \mathrm{U}(1)_{B-L}$. Assuming that these scalars are boundstates of the usual fermions, the following fermionic content reproduces the correct quantum numbers:

$$
\begin{gathered}
\varphi_{i j} \sim \alpha\left(\bar{Q}_{R j} Q_{L i}\right)+\beta\left(\tau_{2} \bar{Q}_{L} Q_{R} \tau_{2}\right)_{i j}+\text { leptonic terms }, \\
\vec{\Delta}_{L} \sim\left(\Psi_{L}^{T} C \tau_{2} \vec{\tau} \Psi_{L}\right), \quad \vec{\Delta}_{R} \sim\left(\Psi_{R}^{T} C \tau_{2} \vec{\tau} \Psi_{R}\right) .
\end{gathered}
$$

Here $Q_{L}, \Psi_{L}\left(Q_{R}, \Psi_{R}\right)$ are left-handed (right-handed) doublets of quarks and leptons, respectively; $i$ and $j$ are isospin indices. In models with composite Higgs bosons generated by four-fermion operators the scalars are, roughly speaking, "square roots" of these fourfermion operators due to the equations of motion. A good starting point to find a set of four-fermion-operators leading to the desired scalars (41) is to square these expressions.

However, the model with composite triplet scalars is not going to be viable: The dynamically generated effective potential is constrained and does not allow the phenomenologically 
required spontaneous parity breaking. The following little exercise will help us to subsequently construct a viable model. Consider spontaneous parity breakdown in left-right models with composite Higgs bosons. It is usually assumed that, in addition to the gauge symmetry, the Lagrangian of the left-right model possesses a discrete parity symmetry under which

$$
Q_{L} \leftrightarrow Q_{R}, \quad \Psi_{L} \leftrightarrow \Psi_{R}, \quad \varphi \leftrightarrow \varphi^{\dagger}, \quad \Delta_{L} \leftrightarrow \Delta_{R}, \quad W_{L} \leftrightarrow W_{R}
$$

Then the $\Delta$-sector of the (radiatively induced) scalar potential contains the terms

$$
\begin{aligned}
V\left(\Delta_{L}, \Delta_{R}\right)= & -m^{2}\left(\Delta_{L}^{\dagger} \Delta_{L}+\Delta_{R}^{\dagger} \Delta_{R}\right) \\
& +\lambda_{1}\left[\left(\Delta_{L}^{\dagger} \Delta_{L}\right)^{2}+\left(\Delta_{R}^{\dagger} \Delta_{R}\right)^{2}\right] \\
& +2 \lambda_{2}\left(\Delta_{L}^{\dagger} \Delta_{L}\right)\left(\Delta_{R}^{\dagger} \Delta_{R}\right)+\ldots,
\end{aligned}
$$

Despite the discrete parity symmetry, parity can be spontaneously broken if $\left\langle\Delta_{R}\right\rangle>$ $\left\langle\Delta_{L}\right\rangle$ [38]. From (45) this can only happen for $\lambda_{2}>\lambda_{1}$, and in the conventional approach $\lambda_{1}$ and $\lambda_{2}$ are chosen appropriately as free parameters of the model. However, the scalar mass terms and couplings in the composite Higgs approach are not arbitrary; they are all calculable in terms of the four-fermion couplings $G_{a}$ and the scale of new physics $\Lambda$ [19. In particular, in the fermion bubble approximation at one loop level the quartic couplings $\lambda_{1}$ and $\lambda_{2}$ are induced through the Majorana-like Yukawa couplings $f\left(\Psi_{L}^{T} C \tau_{2} \vec{\tau} \vec{\Delta}_{L} \Psi_{L}+\Psi_{R}^{T} C \tau_{2} \vec{\tau} \vec{\Delta}_{R} \Psi_{R}\right)+$ h.c., and are given by the diagrams of figure 1 . It

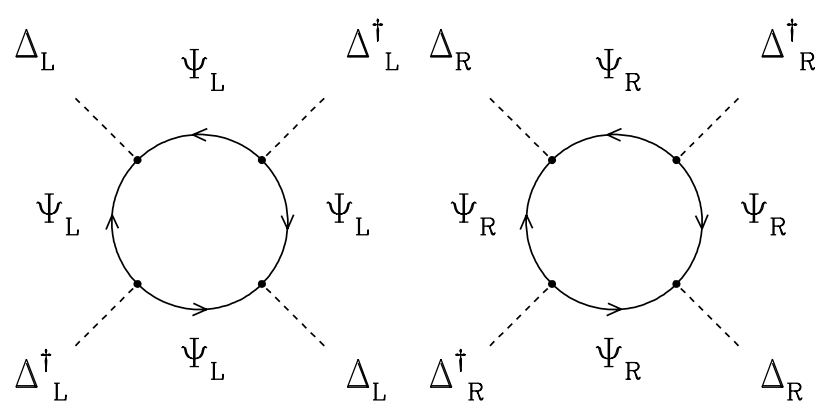

(a)

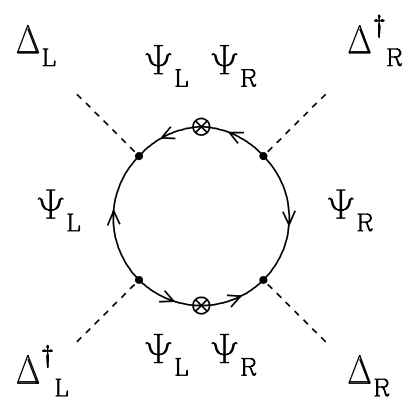

(b)

Figure 1: Fermion loop diagrams contributing to the quartic couplings $\lambda_{1}$ (figure [la) and $\lambda_{2}$ (figure 7 b) for Higgs triplets.

can be seen from figure 1b that to induce the $\lambda_{2}$ term one needs the $\Psi_{L}-\Psi_{R}$ mixing in the fermion line in the loop, i.e. the lepton Dirac mass term insertions. However, the Dirac mass terms are generated, e.g., by the vacuum expectation values of the bi-doublet $\varphi$; they are absent at the parity breaking scale which is supposed to be higher than the electro-weak scale. Even if parity and the electro-weak symmetry are broken simultaneously (which is hardly a phenomenologically viable scenario), this would not save the situation since the diagram of figure $1 \mathrm{~b}$ is finite in the limit $\Lambda \rightarrow \infty$ whereas the one of figure 1a is logarithmically divergent. Consequently, the inequality $\lambda_{2}>\lambda_{1}$ cannot be satisfied. 


\subsection{A Viable Model}

From the above considerations follows that we have to look for a modified low energy scalar sector. Originally left-right-symmetric models were constructed including two doublets [33], $\chi_{L} \sim(2,1,-1)$ and $\chi_{R} \sim(1,2,-1)$. However, these cannot have any gauge invariant interactions with the known quarks and leptons, while the subsequent models with triplets $\Delta_{L}$ and $\Delta_{R}$ are attractive due to Majorana like couplings to leptons and the resulting see-saw mechanism.

In the composite approach the disadvantage of the doublet-model can be turned into a virtue, the only "price" being the introduction of a new gauge singlet fermion. A gauge singlet fermion is required to construct a composite scalar doublet out of fermion bi-linears, since all the known fermions already come in doublets. However, this singlet is quite welcome, since its Majorana like coupling leads to a modified see-saw mechanism [39], which can naturally explain the smallness of neutrino masses. Second, we will see that the chiral singlet fermion in the loops is essential for the correct left-right symmetry breaking pattern to emerge in the composite doublet model.

We therefore assume that in addition to the usual quark and lepton doublets there is a gauge-singlet fermion

$$
S_{L} \sim(1,1,0)
$$

To maintain the discrete parity symmetry one needs a right-handed counterpart of $S_{L}$. This can be either another particle, $S_{R}$, or the right-handed antiparticle of $S_{L},\left(S_{L}\right)^{c} \equiv$ $C \bar{S}_{L}^{T}=S_{R}^{c}$. The latter choice is more economical and at the same time crucial for the $\lambda_{2}$ contribution of the effective potential and the desired symmetry breaking pattern. We therefore assume that under parity operation

$$
S_{L} \leftrightarrow S_{R}^{c} .
$$

With this new singlet and the usual quark and lepton doublets we introduce the following set of gauge-invariant four-fermion interactions:

$$
\begin{array}{r}
\mathcal{L}_{4 f}=G_{1}\left(\bar{Q}_{L i} Q_{R j}\right)\left(\bar{Q}_{R j} Q_{L i}\right)+\left[G_{2}\left(\bar{Q}_{L i} Q_{R j}\right)\left(\tau_{2} \bar{Q}_{L} Q_{R} \tau_{2}\right)_{i j}+\text { h.c. }\right] \\
+G_{3}\left(\bar{\Psi}_{L i} \Psi_{R j}\right)\left(\bar{\Psi}_{R j} \Psi_{L i}\right)+\left[G_{4}\left(\bar{\Psi}_{L i} \Psi_{R j}\right)\left(\tau_{2} \bar{\Psi}_{L} \Psi_{R} \tau_{2}\right)_{i j}+h . c .\right] \\
+\left[G_{5}\left(\bar{Q}_{L i} Q_{R j}\right)\left(\bar{\Psi}_{R j} \Psi_{L i}\right)+h . c .\right]+\left[G_{6}\left(\bar{Q}_{L i} Q_{R j}\right)\left(\tau_{2} \bar{\Psi}_{L} \Psi_{R} \tau_{2}\right)_{i j}+h . c .\right] \\
+G_{7}\left[\left(S_{L}^{T} C \Psi_{L}\right)\left(\bar{\Psi}_{L} C \bar{S}_{L}^{T}\right)+\left(\bar{S}_{L} \Psi_{R}\right)\left(\bar{\Psi}_{R} S_{L}\right)\right]+G_{8}\left(S_{L}^{T} C S_{L}\right)\left(\bar{S}_{L} C \bar{S}_{L}^{T}\right) .
\end{array}
$$

In analogy to the BHL model the $G_{a}$ are dimensionful four-fermion couplings of the order of $\Lambda^{-2}$ motivated by some new physics at $\Lambda$. Notice that the above interactions are not only gauge-invariant, but also (for hermitian $G_{2}, G_{4}, G_{5}$ and $G_{6}$ ) symmetric with respect to the discrete parity operation (42), (47). Note that equation (48) is a rather general ansatz. It will turn out that we need only a subset of the above terms in order to break the symmetries correctly. 
We assume that only the third generation of fermions contribute to $\mathcal{L}_{4 f}$, i.e., deal with a limit where only the heaviest fermions are massive, while all the light fermions are considered to be massless. This appears to be a good starting point from where light fermion masses could, e.g., be generated radiatively. In addition to the bi-doublet $\varphi$ of the structure given in equation (41), the above four-fermion couplings, if critical, can give rise to a pair of composite doublets $\chi_{L}$ and $\chi_{R}$, and also to a singlet scalar $\sigma$ :

$$
\chi_{L} \sim S_{L}^{T} C \Psi_{L}, \quad \chi_{R} \sim \bar{S}_{L} \Psi_{R}=\left(S_{R}^{c}\right)^{T} C \Psi_{R}, \quad \sigma \sim \bar{S}_{L} C \bar{S}_{L}^{T} .
$$

From equations (42) and (47) it follows that under parity we have $\chi_{L} \leftrightarrow \chi_{R}$ and $\sigma \leftrightarrow \sigma^{\dagger}$. Switching to the auxiliary field formalism, the scalars $\chi_{L}, \chi_{R}, \varphi$ and $\sigma$ have the following bare mass terms and Yukawa couplings:

$$
\begin{aligned}
L_{a u x}= & -M_{0}^{2}\left(\chi_{L}^{\dagger} \chi_{L}+\chi_{R}^{\dagger} \chi_{R}\right)-M_{1}^{2} \operatorname{tr}\left(\varphi^{\dagger} \varphi\right) \\
& -\frac{M_{2}^{2}}{2} \operatorname{tr}\left(\varphi^{\dagger} \widetilde{\varphi}+\text { h.c. }\right)-M_{3}^{2} \sigma^{\dagger} \sigma \\
& -\left[\bar{Q}_{L}\left(Y_{1} \varphi+Y_{2} \widetilde{\varphi}\right) Q_{R}+\bar{\Psi}_{L}\left(Y_{3} \varphi+Y_{4} \widetilde{\varphi}\right) \Psi_{R}+\text { h.c. }\right] \\
& -\left[Y_{5}\left(\bar{\Psi}_{L} \chi_{L} S_{R}^{c}+\bar{\Psi}_{R} \chi_{R} S_{L}\right)+Y_{6}\left(S_{L}^{T} C S_{L}\right) \sigma+\text { h.c. }\right],
\end{aligned}
$$

where the field $\widetilde{\varphi} \equiv \tau_{2} \varphi^{*} \tau_{2}$ has the same quantum numbers as $\varphi: \widetilde{\varphi} \sim(2,2,0)$. By integrating out the auxiliary scalar fields one can reproduce the four-fermion structures of equations (48) and express the four-fermion couplings $G_{1}, \ldots, G_{8}$ in terms of the Yukawa couplings $Y_{1}, \ldots, Y_{6}$ and the mass parameters $M_{0}^{2}, M_{1}^{2}, M_{2}^{2}$ and $M_{3}^{2}$ (explicit formulas can be found in [36]). In components, the scalar multiplets of the model are

$$
\begin{array}{cl}
\varphi=\left(\begin{array}{cc}
\varphi_{1}^{0} & \varphi_{2}^{+} \\
\varphi_{1}^{-} & \varphi_{2}^{0}
\end{array}\right), & \langle\varphi\rangle=\left(\begin{array}{cc}
\kappa & 0 \\
0 & \kappa^{\prime}
\end{array}\right), \\
\chi_{L}=\left(\begin{array}{c}
\chi_{L}^{0} \\
\chi_{L}^{L}
\end{array}\right), & \chi_{R}=\left(\begin{array}{c}
\chi_{R}^{0} \\
\chi_{R}^{-}
\end{array}\right) .
\end{array}
$$

Let us now consider parity breaking in the present left-right model. In a viable scenario the $\mathrm{SU}(2)_{R}$ symmetry should be broken at the right-handed scale $\mu_{R}$ by $\left\langle\chi_{R}^{0}\right\rangle=v_{R}$, and the electro-weak symmetry has to be broken at $\mu_{E W}$ by the vacuum expectation values of $\varphi$ and possibly of $\chi_{L}^{0}\left(\equiv v_{L}\right)$. Using the Yukawa couplings of the doublets $\chi_{L}$ and $\chi_{R}$ [see equation (50)], one can calculate the fermion-loop contributions to the quartic couplings $\lambda_{1}\left[\left(\chi_{L}^{\dagger} \chi_{L}\right)^{2}+\left(\chi_{R}^{\dagger} \chi_{R}\right)^{2}\right]$ and $2 \lambda_{2}\left(\chi_{L}^{\dagger} \chi_{L}\right)\left(\chi_{R}^{\dagger} \chi_{R}\right)$ in the effective Higgs potential (figures $2 \mathrm{a}$ and $2 \mathrm{~b}$ ). Unlike in the triplet case, the $\lambda_{1}$ and $\lambda_{2}$ terms are now given by similar diagrams, because the gauge singlet couples both to $\chi_{L}$ and $\chi_{R}$. Since the Yukawa couplings of $\chi_{L}$ and $\chi_{R}$ coincide (which is just the consequence of the discrete parity symmetry), figures $2 \mathrm{a}$ and $2 \mathrm{~b}$ yield $\lambda_{1}=\lambda_{2}$. Recall that one needs $\lambda_{2}>\lambda_{1}$ to have spontaneous parity breakdown, and thus the situation here has already improved compared to the triplet scenario. As we shall see, taking into account the gauge boson loop contributions to $\lambda_{1}$ and $\lambda_{2}$ will 


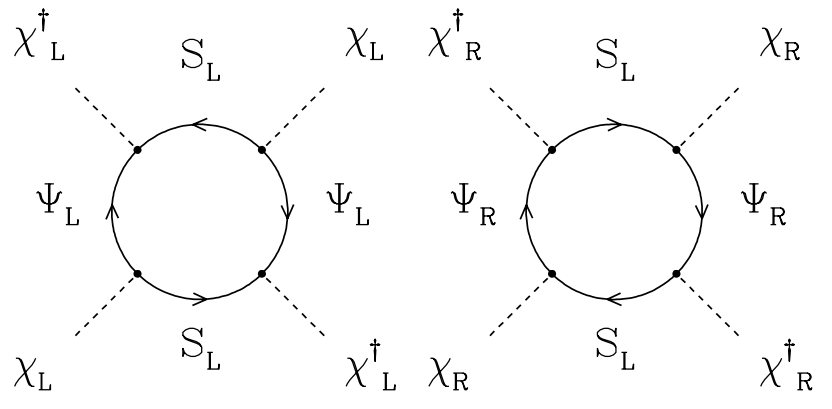

(a)

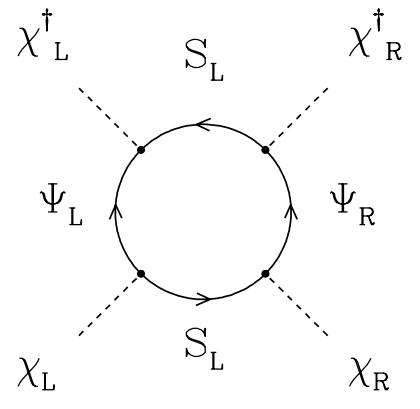

(b)

Figure 2: Fermion loop diagrams contributing to the quartic couplings $\lambda_{1}$ (figure 2 a) and $\lambda_{2}$ (figure (ab) for the Higgs doublets $\chi_{L / R}$.

automatically secure this relation: Both $\lambda_{1}$ and $\lambda_{2}$ obtain corrections from $\mathrm{U}(1)_{B-L}$ gauge boson loops, whereas only $\lambda_{1}$ is corrected by diagrams with $W_{L}^{i}$ or $W_{R}^{i}$ loops (see figure 3 ). Since all these contributions have a relative minus sign compared to the fermion loop ones,

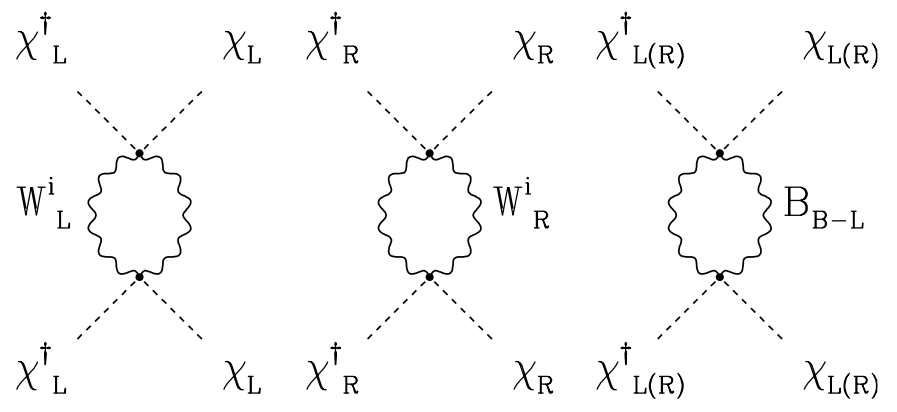

(a)

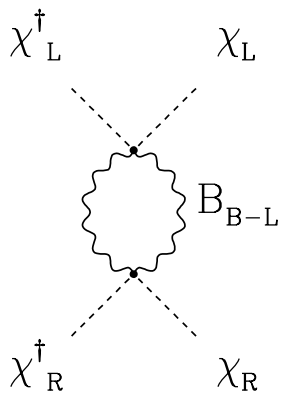

(b)

Figure 3: Gauge boson loop diagrams contributing to the quartic couplings $\lambda_{1}$ (figure Ba) and $\lambda_{2}$ (figure $3 \mathrm{~b}$ ) for the Higgs doublets $\chi_{L / R}$ in Landau gauge.

one finds $\lambda_{2}>\lambda_{1}$ irrespective of the values of the Yukawa or gauge couplings or any other parameter of the model, provided that the $\mathrm{SU}(2)$ gauge coupling $g_{2} \neq 0$ [compare the expressions for $\lambda_{1}$ and $\lambda_{2}$ in (61) below]. Thus the condition for spontaneous parity breakdown is automatically satisfied in the model.

We have a very interesting situation here. In a model with composite triplets $\Delta_{L}$ and $\Delta_{R}$ parity is never broken, i.e. the model is not phenomenologically viable. At the same time, in the model with two composite doublets $\chi_{L}$ and $\chi_{R}$ instead of two triplets (which requires introduction of an additional singlet fermion $S_{L}$ ) parity is broken automatically. This means that, unlike in conventional left-right models, in the composite Higgs approach whether or not parity can be spontaneously broken depends on the particle content of the model rather than on the choice of the parameters of the Higgs potential. 
As soon as we know the vacuum structure of the model (see below), i.e. the different vacuum expectation values for composite scalar fields, equation (50) yields the expressions for the resulting fermion masses. The masses of the quarks and charged leptons and the Dirac neutrino mass $m_{D}$ are given by the vacuum expectation values of the bi-doublet ${ }^{9}$ :

$$
\begin{gathered}
m_{t}=Y_{1} \kappa+Y_{2} \kappa^{\prime}, \quad m_{D}=Y_{3} \kappa+Y_{4} \kappa^{\prime}, \\
m_{b}=Y_{1} \kappa^{\prime}+Y_{2} \kappa, \quad m_{\tau}=Y_{3} \kappa^{\prime}+Y_{4} \kappa .
\end{gathered}
$$

It is well known that left-right-symmetric models with only doublet Higgs scalars can usually not explain small neutrino masses in a natural way. As pointed out earlier, introducing the singlet fermion $S_{L}$ not only provides the spontaneous parity breaking in the composite model, but also cures the neutrino mass problem. It was first noticed by Wolfenstein and Wyler [39] that with an additional singlet neutral fermion $S_{L}$ the neutrino mass matrix takes the form (in the basis $\left(\nu_{L}, \nu_{L}^{c}, S_{L}\right)$ )

$$
M_{\nu}=\left(\begin{array}{ccc}
0 & m_{D} & \beta \\
m_{D} & 0 & M_{\nu_{R}} \\
\beta & M_{\nu_{R}} & \widetilde{\mu}
\end{array}\right),
$$

where the entries $\beta, M_{\nu_{R}}$ and $\widetilde{\mu}$ can be read off from equation (50),

$$
\beta=Y_{5} v_{L}, \quad M_{\nu_{R}}=Y_{5} v_{R} \quad \tilde{\mu}=2 Y_{6} \sigma_{0},
$$

with $\sigma_{0} \equiv\langle\sigma\rangle$. For $v_{R} \gg \kappa, \kappa^{\prime}, v_{L}$ and $v_{R} \gtrsim \sigma_{0}$ one obtains two heavy Majorana neutrino mass eigenstates with the masses $\sim M_{\nu_{R}}$ and a light Majorana neutrino with the mass $m_{\nu} \simeq \widetilde{\mu}\left(m_{D}^{2} / M_{\nu_{R}}^{2}\right)-2 \beta m_{D} / M_{\nu_{R}}$ which vanishes in the limit $M_{\nu_{R}} \rightarrow \infty$. This is the modified seesaw mechanism which provides the smallness of neutrino mass.

\subsection{Effective Potential and Vacuum Structure}

The rough analysis of parity breakdown given above demonstrated that this model can in principle work. Now the next step is to calculate the full low energy effective potential of the theory, analyse the vacuum structure and the resulting patterns of spontaneous symmetry breaking. As we have already seen, this is a nontrivial issue since the potential parameters are not free but constrained and calculable in terms of the initial 6 Yukawa couplings, or four-fermion-terms, respectively.

The effective potential arises as part of a complete renormalizable low energy effective Lagrangian which emerges after integrating out the high energy degrees of freedom. Explicitly, one analyses the scalar two- and four-point functions and finds the effective Lagrangian

$$
\mathcal{L}_{\text {eff }}=\mathcal{L}_{0}+\mathcal{L}_{\text {Yuk }}+Z_{\varphi} \operatorname{tr}\left[\left(D^{\mu} \varphi\right)^{\dagger}\left(D_{\mu} \varphi\right)\right]+Z_{\sigma}\left(\partial^{\mu} \sigma\right)^{\dagger}\left(\partial_{\mu} \sigma\right)
$$

\footnotetext{
${ }^{9} \mathrm{We}$ assume all the vacuum expectation values to be real.
} 


$$
\begin{aligned}
& +Z_{\chi}\left[\left(D^{\mu} \chi_{L}\right)^{\dagger}\left(D_{\mu} \chi_{L}\right)+\left(D^{\mu} \chi_{R}\right)^{\dagger}\left(D_{\mu} \chi_{R}\right)\right] \\
& +V_{\text {eff }}
\end{aligned}
$$

$\mathcal{L}_{0}$ contains the gauge-invariant kinetic terms of fermions and gauge bosons, while $\mathcal{L}_{\text {Yuk }}$ represents the Yukawa-coupling terms in equation (50). The additional terms consist of mass corrections, gauge invariant kinetic terms for the scalars and the full effective scalar potential. The effective Higgs potential $V_{\text {eff }}$ in equation (56) reads

$$
\begin{aligned}
V_{\text {eff }}= & \widetilde{M}_{0}^{2}\left(\chi_{L}^{\dagger} \chi_{L}+\chi_{R}^{\dagger} \chi_{R}\right)+\widetilde{M}_{1}^{2} \operatorname{tr}\left(\varphi^{\dagger} \varphi\right)+\frac{\widetilde{M}_{2}^{2}}{2} \operatorname{tr}\left(\varphi^{\dagger} \widetilde{\varphi}+h . c .\right)+\widetilde{M}_{3}^{2} \sigma^{\dagger} \sigma \\
& +\lambda_{1}\left[\left(\chi_{L}^{\dagger} \chi_{L}\right)^{2}+\left(\chi_{R}^{\dagger} \chi_{R}\right)^{2}\right]+2 \lambda_{2}\left(\chi_{L}^{\dagger} \chi_{L}\right)\left(\chi_{R}^{\dagger} \chi_{R}\right) \\
& +\frac{1}{2} \lambda_{3}\left[\chi_{L}^{\dagger}\left(Y_{3} \varphi+Y_{4} \widetilde{\varphi}\right) \chi_{R} \sigma^{\dagger}+h . c .\right] \\
& +\lambda_{4}\left[\chi_{L}^{\dagger}\left(Y_{3} \varphi+Y_{4} \widetilde{\varphi}\right)\left(Y_{3} \varphi^{\dagger}+Y_{4} \widetilde{\varphi}^{\dagger}\right) \chi_{L}\right. \\
& \left.\quad+\chi_{R}^{\dagger}\left(Y_{3} \varphi^{\dagger}+Y_{4} \widetilde{\varphi}^{\dagger}\right)\left(Y_{3} \varphi+Y_{4} \widetilde{\varphi}\right) \chi_{R}\right] \\
& +\lambda_{5}\left(\chi_{L}^{\dagger} \chi_{L}+\chi_{R}^{\dagger} \chi_{R}\right) \operatorname{tr}\left(\varphi^{\dagger} \varphi\right)+\lambda_{6}\left(\chi_{L}^{\dagger} \chi_{L}+\chi_{R}^{\dagger} \chi_{R}\right) \sigma^{\dagger} \sigma \\
& +\lambda_{7}^{\prime} \operatorname{tr}\left(\varphi^{\dagger} \varphi \varphi^{\dagger} \varphi\right)+\frac{1}{3} \lambda_{8}^{\prime} \operatorname{tr}\left(\varphi^{\dagger} \widetilde{\varphi} \widetilde{\varphi}^{\dagger} \varphi\right)+\frac{1}{12} \lambda_{8}^{\prime}\left[\operatorname{tr}\left(\varphi^{\dagger} \widetilde{\varphi} \varphi^{\dagger} \widetilde{\varphi}\right)+h . c .\right] \\
& +\frac{1}{2} \lambda_{9}\left[\operatorname{tr}\left(\varphi^{\dagger} \varphi \varphi^{\dagger} \widetilde{\varphi}\right)+h . c .\right]+\lambda_{0}\left[\operatorname{tr}\left(\varphi^{\dagger} \varphi\right)\right]^{2}+\lambda_{10}\left(\sigma^{\dagger} \sigma\right)^{2},
\end{aligned}
$$

with the mass term corrections and quartic couplings $\lambda_{i}$ given as functions of Yukawa couplings and the cutoff. For our purposes we need only the following expressions for the potential parameters:

$$
\begin{aligned}
\widetilde{M}_{0}^{2} & =M_{0}^{2}-\frac{1}{8 \pi^{2}}\left[Y_{5}^{2}-\frac{3}{8} Z_{\chi}\left(3 g_{2}^{2}+g_{1}^{2}\right)\right]\left(\Lambda^{2}-\mu^{2}\right) \\
\widetilde{M}_{1}^{2} & =M_{0}^{2}-\frac{1}{8 \pi^{2}}\left\{\left[N_{c}\left(Y_{1}^{2}+Y_{2}^{2}\right)+\left(Y_{3}^{2}+Y_{4}^{2}\right)\right]-\frac{9}{4} Z_{\varphi} g_{2}^{2}\right\}\left(\Lambda^{2}-\mu^{2}\right) \\
\widetilde{M}_{2}^{2} & =M_{2}^{2}-\frac{1}{4 \pi^{2}}\left(N_{c} Y_{1} Y_{2}+Y_{3} Y_{4}\right)\left(\Lambda^{2}-\mu^{2}\right), \\
\widetilde{M}_{3}^{2} & =M_{0}^{2}-\frac{1}{4 \pi^{2}} Y_{6}^{2}\left(\Lambda^{2}-\mu^{2}\right) \\
\lambda_{1} & =\frac{1}{16 \pi^{2}}\left[Y_{5}^{4}-\frac{3}{16}\left(3 g_{2}^{4}+2 g_{2}^{2} g_{1}^{2}+g_{1}^{4}\right) Z_{\chi}^{2}\right] \ln \left(\frac{\Lambda^{2}}{\mu^{2}}\right) \\
\lambda_{2} & =\frac{1}{16 \pi^{2}}\left[Y_{5}^{4}-\frac{3}{16} g_{1}^{4} Z_{\chi}^{2}\right] \ln \left(\frac{\Lambda^{2}}{\mu^{2}}\right)
\end{aligned}
$$




$$
\begin{aligned}
& \lambda_{0}=\frac{1}{16 \pi^{2}}\left[-\frac{3}{2} g_{2}^{4} Z_{\varphi}^{2}\right] \ln \left(\frac{\Lambda^{2}}{\mu^{2}}\right) \\
& \lambda_{5}=\frac{1}{16 \pi^{2}}\left[-\frac{9}{8} g_{2}^{4} Z_{\varphi} Z_{\chi}\right] \ln \left(\frac{\Lambda^{2}}{\mu^{2}}\right), \\
& \lambda_{7}^{\prime}=\frac{1}{16 \pi^{2}}\left[N_{c}\left(Y_{1}^{4}+Y_{2}^{4}\right)+\left(Y_{3}^{4}+Y_{4}^{4}\right)\right] \ln \left(\frac{\Lambda^{2}}{\mu^{2}}\right) \\
& \lambda_{7}=\lambda_{7}^{\prime}+\lambda_{0} .
\end{aligned}
$$

Here $g_{2}$ and $g_{1}$ are the $\mathrm{SU}(2)$ and $\mathrm{U}(1)_{B-L}$ gauge couplings, respectively. The scalar wavefunction renormalization constants are given by

$$
\begin{aligned}
& Z_{\varphi}=\frac{1}{16 \pi^{2}}\left[N_{c}\left(Y_{1}^{2}+Y_{2}^{2}\right)+Y_{3}^{2}+Y_{4}^{2}\right] \ln \left(\frac{\Lambda^{2}}{\mu^{2}}\right) \\
& Z_{\chi}=\frac{1}{16 \pi^{2}} Y_{5}^{2} \ln \left(\frac{\Lambda^{2}}{\mu^{2}}\right), \quad Z_{\sigma}=\frac{1}{16 \pi^{2}} 2 Y_{6}^{2} \ln \left(\frac{\Lambda^{2}}{\mu^{2}}\right) .
\end{aligned}
$$

The above parameters are running parameters, depending on the energy scale $\mu$, and parametrize the effective Lagrangian at that energy scale 10 . At $\mu \rightarrow \Lambda$ the kinetic terms and quartic couplings of the scalar fields vanish, their mass terms are driven towards their bare values, and one recovers the Lagrangian with auxiliary static scalar fields.

Spontaneous symmetry breaking generally occurs when scalar mass terms become negative, in the presence of quartic scalar interactions. While the bare mass parameters $M_{i}^{2}$ in equation (50) are positive, the corresponding running quantities $\widetilde{M}_{i}^{2}$, given by equations (57) - (60), may become negative at low energy scales provided that the corresponding Yukawa couplings are large enough. Those values for which this occurs at $\mu=0$ we shall call the critical Yukawa couplings. Of course, in 4F-language this correspond to the critical values of the original $4 \mathrm{~F}$-couplings. For $\widetilde{M}_{i}^{2}$ to become negative at some scale $\mu^{2}>0$ the corresponding Yukawa couplings, or combinations of them, must be above their critical values. If this is to happen at scales $\mu \ll \Lambda$ the Yukawa couplings must be fine-tuned very closely to their critical values to ensure the proper cancellation between the large bare masses of the scalars and the $\Lambda^{2}$ corrections in equations (57) - (60). This is equivalent to the usual fine-tuning problem of gauge theories with elementary Higgs scalars [19].

We assume that the scale $\mu_{R}$ at which parity gets spontaneously broken (i.e. $\chi_{R}^{0}$ develops a vacuum expectation value) is higher than the electro-weak scale $\mu_{E W} \sim 100 \mathrm{GeV}$, i.e. that $\widetilde{M}_{0}^{2}$ changes its sign at a higher scale than $\widetilde{M}_{1}^{2}$. This means that $Y_{5}^{2}-(3 / 8) Z_{\chi}\left(3 g_{2}^{2}+g_{1}^{2}\right)$ should be bigger than $\tilde{Y}^{2}-\frac{9}{4} Z_{\varphi} g_{2}^{2}$ [see equations (57) and (58)], where $\tilde{Y}^{2} \equiv N_{c}\left(Y_{1}^{2}+Y_{2}^{2}\right)+$ $\left(Y_{3}^{2}+Y_{4}^{2}\right)$. By analysing the minima of the effective potential (56) one can show that if

\footnotetext{
${ }^{10}$ This bubble-approximation running exactly coincides with the running one would get from 1-loop renormalization group equations keeping only trace terms in the relevant $\beta$ functions and imposing the compositeness boundary conditions [19].
} 
the condition

$$
Y_{5}^{2}-\frac{3}{8} Z_{\chi}\left(3 g_{2}^{2}+g_{1}^{2}\right)>2 Y_{6}^{2}
$$

is satisfied, either $\chi_{R}$ or $\chi_{L}$ (but not both) acquire a vacuum expectation value but the $\sigma$ field does not, whereas for the opposite condition $\sigma$ acquires a non-zero vacuum expectation value, but not $\chi_{R}$ or $\chi_{L}$. Clearly the latter situation is phenomenologically unacceptable, but by choosing the four-fermion couplings $G_{7}$ and $G_{8}$ accordingly we can easily satisfy equation (63).

\subsubsection{The Case $\kappa^{\prime}=0$}

Let us now discuss the vacuum structure below the electro-weak breaking scale. The nonvanishing vacuum expectation values are $v_{R}, \kappa$ and $\kappa^{\prime}$. Since $m_{t} \gg m_{b}$, it follows from equation (52) that $\kappa$ should be much larger than $\kappa^{\prime}$ or vice versa provided no significant cancellation between $Y_{1} \kappa^{\prime}$ and $Y_{2} \kappa$ occurs. Without loss of generality one can take $\kappa>\kappa^{\prime}$. To further simplify the discussion, we will first consider the frequently used assumption 40. $\kappa^{\prime}=0$. The relation $m_{t} \gg m_{b}$ then translates into $Y_{1} \gg Y_{2}$. In the conventional approach this assumption does not lead to any contradiction with phenomenology. However, as we shall see, in the composite model the condition $\kappa^{\prime}=0$ cannot be exact.

Consistency of the first-derivative conditions with $\kappa^{\prime}=0$ requires $Y_{1} Y_{2}=0, Y_{3} Y_{4}=0$ and $M_{2}^{2}=0$ (this gives $\widetilde{M}_{2}^{2}=\lambda_{9}=0$, and as follows from equation (56), all the terms in the effective potential which are linear in $\kappa^{\prime}$ become zero in this limit, as they should). The condition $Y_{1} Y_{2}=0$ along with $\kappa^{\prime}=0$ implies that either $Y_{1}=0, m_{t}=0$ or $Y_{2}=0$, $m_{b}=0$. The first possibility is obviously phenomenologically unacceptable, whereas the second one can be considered as a reasonable first approximation. Therefore we assume $Y_{1} \neq 0$ and $Y_{2}=0$. The situation is less clear for the lepton Yukawa couplings $Y_{3}$ and $Y_{4}$. Since $m_{\tau} \ll m_{t}$ and the Dirac mass $m_{D}$ of $\nu_{\tau}$ is unknown, one can choose either $Y_{3} \neq 0$, $Y_{4}=0$ or $Y_{3}=0, Y_{4} \neq 0$. It turns out that the vacuum stability condition in this model requires $Y_{4}^{2}>Y_{3}^{2}$, therefore we choose $Y_{3}=0$ and $Y_{4} \neq 0$.

For $\sigma_{0}=v_{L}=\kappa^{\prime}=Y_{2}=Y_{3}=0$ one can easily find expressions for the vacuum expectation values of $\chi_{R}$ and $\varphi$. Approximate expressions in terms of the parity breaking scale $\mu_{R}$ and the electro-weak breaking scale $\mu_{E W}$ are

$$
v_{R}^{2} \simeq\left(\frac{M_{0}^{2}}{\Lambda^{2}}\right) \frac{\mu_{R}^{2}}{2 \lambda_{1}}, \quad \kappa^{2} \simeq\left(\frac{M_{0}^{2}}{\Lambda^{2}}\right) \frac{\mu_{E W}^{2}}{2 \lambda_{7}},
$$

and the ratio of the squared vacuum expectation values can be written as

$$
\frac{\kappa^{2}}{v_{R}^{2}} \simeq\left(\frac{\lambda_{1}}{\lambda_{7}}\right) \frac{\mu_{E W}^{2}}{\mu_{R}^{2}} \sim \frac{\mu_{E W}^{2}}{\mu_{R}^{2}} \simeq \frac{\left|\lambda_{5}\right|}{2 \lambda_{1}}+\frac{\mu_{1}^{2}}{\mu_{R}^{2}} .
$$

The parity breaking scale $\mu_{R}$ is the scale where the effective mass term $\widetilde{M}_{0}^{2}$ becomes negative for a given Yukawa coupling $Y_{5}>\left(Y_{5}\right)_{\text {crit }}$ (formally $\mu_{R}^{2}<0$ for sub-critical $Y_{5}$ ), 
while $\mu_{1}$ is the scale, different from $\mu_{E W}$, where this happens for the mass term $\widetilde{M}_{1}^{2}$ and a given $\tilde{Y}^{2}$.

Recall now that in conventional left-right models with $\mu_{E W} \ll \mu_{R} \ll \Lambda_{G U T}$ (or $\Lambda_{\text {Planck }}$ ) one has to fine-tune two gauge hierarchies: $\Lambda_{G U T}-\mu_{R}$ and $\mu_{R}-\mu_{E W}$. The situation here is quite similar: to achieve $\mu_{E W} \ll \mu_{R} \ll \Lambda$ one has to fine-tune two Yukawa couplings, $Y_{5}^{2}$ and $\tilde{Y}^{2}$. Tuning of $Y_{5}^{2}$ allows for the hierarchy $\mu_{R}^{2} \ll \Lambda^{2}$; one then needs to adjust $\widetilde{Y}^{2}$ (or $\mu_{1}^{2}$ ) to achieve $\mu_{E W}^{2} \ll \mu_{R}^{2}$ through equation (65).

Since $\lambda_{5}$ only contains relatively small gauge couplings while $Y_{5} \sim \mathcal{O}(1)$, we typically have $\left|\lambda_{5}\right| /\left(2 \lambda_{1}\right) \sim 10^{-2}$. Thus, if there is no significant cancellation between the two terms in (65), one obtains a right-handed scale of the order of a few $\mathrm{TeV}$. Unfortunately, such a low left-right scale scenario is not viable. As we shall see below, the squared masses of two Higgs bosons become negative (i.e. the vacuum becomes unstable) unless $v_{R} \gtrsim 20 \mathrm{TeV}$. This requires some cancellation $\square$ in equation (65), and then the right-handed scale $v_{R} \sim \mu_{R}$ can in principle lie anywhere between a few tens of $\mathrm{TeV}$ and $\Lambda$. However, if one prefers "minimal cancellation" in equation (65), by about two orders of magnitude or so, one would arrive at a value of $v_{R}$ around $20 \mathrm{TeV}$. In any case it is interesting that the partial cancellation of the two terms in (65) implies $\mu_{1}^{2}<0$, i.e. that $\tilde{Y}^{2}$ must be below its critical value. This means that $\widetilde{M}_{1}^{2}$ never becomes negative. In fact it is the $\widetilde{M}_{0}^{2}$ term, responsible for parity breakdown, that also drives the vacuum expectation value of the bi-doublet. It follows from the condition $\partial V_{\text {eff }} / \partial \kappa=0$ that the effective driving term for $\kappa$ is $\widetilde{M}_{1}^{2}+\lambda_{5} v_{R}^{2}$; it may become negative for large enough $v_{R}^{2}$ even if $\widetilde{M}_{1}^{2}$ is positive (remember that $\lambda_{5}<0$ ). Thus one finds a tumbling scenario where the breakdown of parity and $\mathrm{SU}(2)_{R}$ occurring at the scale $\mu_{R}$ causes the breakdown of the electro-weak symmetry at a lower scale $\mu_{E W}$.

\subsubsection{The Case $\kappa^{\prime} \neq 0$}

After having established the vacuum structure for the simplified case $v_{R}, \kappa \neq 0, \kappa^{\prime}=0$, we now turn to the more general case with $\kappa^{\prime} \neq 0$. Under the assumption that the righthanded scale is sufficiently above the electro-weak scale, the details of parity breakdown should remain unchanged. Analysing the minimum conditions in the $\kappa-\kappa^{\prime}$-sector, one finds that the ratio of vacuum expectation values is approximately given by the ratio of lepton Yukawa couplings:

$$
\frac{\kappa^{\prime}}{\kappa}=-\frac{Y_{3}}{Y_{4}}+\mathcal{O}\left(\frac{\kappa^{2}}{v_{R}^{2}}\right) .
$$

Provided the condition (63) is fulfilled, one can show [36] that $v_{L}$ and $\sigma_{0}$ also remain zero after electro-weak symmetry breaking. The diagonalization of the scalar mass matrices leads to positive values for the squared masses, except zero for the expected Goldstone bosons, and thus the described situation is a true minimum of the model.

\footnotetext{
${ }^{11}$ Notice that this does not increase the number of the parameters to be tuned but just shifts the value to which one of them should be adjusted.
} 


\subsection{Fermion Masses}

Now we turn to the calculation of fermion and scalar masses, first in the simplified limit. To calculate physical observables one should first rescale the Higgs fields by absorbing the $Z$-factors in equation (56) into the definitions of the scalar fields to bring their kinetic terms into the canonical form. This amounts to dividing the (mass) ${ }^{2}$ terms by the corresponding $Z$-factors, Yukawa couplings by $\sqrt{Z}$ and multiplying the scalar fields and their vacuum expectation values by $\sqrt{Z}$. Renormalization factors of the quartic couplings depend on the scalars involved and can be readily read off from the effective potential. We will use hats $\left(^{\wedge}\right)$ to denote quantities in the new normalization.

As pointed out earlier, the minimization of the effective Higgs potential gives $\sigma_{0}=$ $0=v_{L}$. This means that the entries $\beta$ and $\widetilde{\mu}$ in the neutrino mass matrix (53) are zero. As a result one finds an exactly massless neutrino eigenstate and two heavy Majorana neutrinos with degenerate masses $\sqrt{M_{\nu_{R}}^{2}+m_{D}^{2}}$ and opposite CP-parities which combine to form a heavy Dirac neutrino. Since $m_{D} \ll M_{\nu_{R}}$ the electro-weak eigenstate $\nu_{L} \equiv \nu_{\tau}$ is predominantly the massless eigenstate, whereas the right-handed neutrino $\nu_{R}$ and the singlet fermion $S_{L}$ consists predominantly of the heavy eigenstates.

As mentioned before, for $\kappa^{\prime}=0$ we have $Y_{2}=0=Y_{3}$. This yields $m_{t}=Y_{1} \kappa, m_{\tau}=Y_{4} \kappa$ and $m_{b}=m_{D}=0$. Vanishing Dirac neutrino mass $m_{D}$ implies the absence of neutrino mixing, and the heavy neutrino mass is now $M_{\nu_{R}}=Y_{5} v_{R}$. From equations (52) and (52) and the definition of the renormalized Yukawa couplings one can readily find

$$
\begin{aligned}
\hat{\kappa}^{2} & =(174 \mathrm{GeV})^{2} \\
& =N_{c} m_{t}^{2}\left(1+\frac{Y_{4}^{2}}{N_{c} Y_{1}^{2}}\right) \frac{1}{16 \pi^{2}} \ln \left(\frac{\Lambda^{2}}{\mu^{2}}\right) \\
& \approx \frac{m_{t}^{2} N_{c}}{16 \pi^{2}} \ln \left(\frac{\Lambda^{2}}{\mu^{2}}\right) \equiv m_{t}^{2} N_{c} l_{0} .
\end{aligned}
$$

Here $\hat{\kappa}$ (or $\sqrt{\hat{\kappa}^{2}+\hat{\kappa}^{\prime 2}}$ for $\kappa^{\prime} \neq 0$ ) is identified with the usual electro-weak vacuum expectation value and the top quark mass is fixed in terms of this known vacuum expectation value and the scale of new physics $\Lambda$. Note that this expression coincides with the one derived in bubble approximation by BHL [19]. From equation (67) it follows that the top quark mass depends on $\Lambda$ logarithmically. For example, for $\Lambda=10^{15} \mathrm{GeV}$ one finds $m_{t} \simeq 165 \mathrm{GeV}$. However, as in the BHL-model, the renormalization group analysis will change this result considerably.

Using considerations similar to those which led to equation (67), it is easy to obtain the following relation between the right-handed vacuum expectation value $v_{R}$, the heavy neutrino mass $M_{\nu_{R}}$ and the scale $\Lambda$ :

$$
\hat{v}_{R}^{2}=M_{\nu_{R}}^{2} \frac{1}{16 \pi^{2}} \ln \left(\frac{\Lambda^{2}}{\mu^{2}}\right)=M_{\nu_{R}}^{2} \cdot l_{0} .
$$


Note that $\mu \approx m_{t}$ is understood in equation (67), whereas $\mu \approx M_{\nu_{R}}$ in equation (68). We will assume $m_{t}, M_{\nu_{R}} \ll \Lambda$ and $M_{\nu_{R}} / m_{t} \ll \Lambda / M_{\nu_{R}}$ throughout this article, therefore $\ln \frac{\Lambda^{2}}{m_{t}^{2}} \approx \ln \frac{\Lambda^{2}}{M_{\nu_{R}}^{2}}$, i.e. the logarithmic factor $l_{0}$ is universal. Then from equations (67) and (68) one finds

$$
\frac{\hat{v}_{R}^{2}}{M_{\nu_{R}}^{2}} \approx \frac{1}{3} \frac{\hat{\kappa}^{2}}{m_{t}^{2}}
$$

Notice that the central top mass value from the Fermilab collaborations 41, $m_{t}=175 \mathrm{GeV}$, implies $l_{0} \approx 1 / 3$. The mass of the $\tau$ lepton is not predicted in this model since it is only weakly coupled to the bi-doublet; it is given by $m_{\tau}=\left(Y_{4} / Y_{1}\right) m_{t}$ and can be adjusted to a desirable value by choosing the proper magnitude of the ratio $Y_{4} / Y_{1}$, or $G_{3} / G_{1}$.

The composite Higgs bosons in this model include the would-be Goldstone bosons $G_{1}^{ \pm} \approx$ $\chi_{R}^{ \pm}$(eaten by $W_{R}^{ \pm}$), $G_{2}^{ \pm}=\varphi_{1}^{ \pm}$(eaten by $\left.W_{L}^{ \pm}\right), G_{1}^{0}=\chi_{R i}^{0}$ (eaten by $Z_{R}$ ) and $G_{2}^{0}=\varphi_{1 i}^{0}$ (eaten by $Z_{L}$ ). The physical Higgs boson sector of the model contains two CP-even neutral scalars $H_{1}^{0} \approx \chi_{R r}^{0}$ and $H_{2}^{0} \approx \varphi_{1 r}^{0}$ with the masses

$$
\begin{aligned}
& M_{H_{1}^{0}}^{2} \simeq 4 M_{\nu_{R}}^{2}\left[1-\frac{3}{16}\left(3 g^{4}+2 g^{2} g^{\prime 2}+g^{\prime 4}\right) l_{0}^{2}\right] \approx 4 M_{\nu_{R}}^{2}, \\
& M_{H_{2}^{0}}^{2} \simeq 4 m_{t}^{2}\left(1-\frac{m_{\tau}^{2}}{3 m_{t}^{2}}-\frac{9}{4} g^{4} l_{0}^{2}\right) \approx 4 m_{t}^{2},
\end{aligned}
$$

which are directly related to the two steps of symmetry breaking, $\mathrm{SU}(2)_{R} \times \mathrm{U}(1)_{B-L} \rightarrow$ $\mathrm{U}(1)_{Y}$ and $\mathrm{SU}(2)_{L} \times \mathrm{U}(1)_{Y} \rightarrow \mathrm{U}(1)_{e m}$. The mass of the scalar $H_{2}^{0}$, which is the analog of the Standard Model Higgs boson [equation (71)], essentially coincides with the one obtained in the bubble approximation by BHL [19]. This simply reflects the fact that this boson is the $t \bar{t}$ bound-state with a mass of $\approx 2 m_{t}$. Analogously, the mass of the heavy CP-even scalar $H_{1}^{0} \approx \chi_{R r}^{0}$ is approximately $2 M_{\nu_{R}}$ since it is a bound-state of heavy neutrinos.

Further, there are the charged Higgs bosons $H_{3}^{ \pm} \approx \varphi_{2}^{ \pm}$with their neutral CP-even and CP-odd partners $H_{3 r}^{0}=\varphi_{2 r}^{0}$ and $H_{3 i}^{0}=\varphi_{2 i}^{0}$, and finally the $\chi_{L}$-fields $H_{4}^{ \pm}=\chi_{L}^{ \pm}, H_{4 r}^{0}=\chi_{L r}^{0}$ and $H_{4 i}^{0}=\chi_{L i}^{0}$ with the masses

$$
\begin{aligned}
& M_{H_{3}^{ \pm}}^{2} \approx \frac{2}{3} M_{\nu_{R}}^{2} \frac{m_{\tau}^{2}}{m_{t}^{2}}, \\
& M_{H_{3 r}^{0}}^{2}=M_{H_{3 i}^{0}}^{2} \approx \frac{2}{3} M_{\nu_{R}}^{2} \frac{m_{\tau}^{2}}{m_{t}^{2}}-\frac{1}{2} M_{H_{2}^{0}}^{2}, \\
& M_{H_{4}^{ \pm}}^{2}=\frac{3}{8}\left(3 g_{2}^{4}+2 g_{2}^{2} g_{1}^{2}\right) l_{0}^{2} M_{\nu_{R}}^{2}+2 m_{\tau}^{2} \\
& M_{H_{4 r}^{0}}^{2}=M_{H_{4 i}^{0}}^{2}=\frac{3}{8}\left(3 g_{2}^{4}+2 g_{2}^{2} g_{1}^{2}\right) l_{0}^{2} M_{\nu_{R}}^{2} .
\end{aligned}
$$

In conventional left-right models only one scalar, which is the analog of the Standard Model Higgs boson, is light (at the electro-weak scale), all the others have their masses of the order 
of the right-handed scale $M_{\nu_{R}}$ [33, 34, 40, 42]. In this composite case, the masses of those scalars are also proportional to $M_{\nu_{R}}$, but all of them except the mass of $H_{1}^{0}$ have some suppression factors. The mass of the charged scalars $H_{3}^{ \pm} \approx \varphi_{2}^{ \pm}$is suppressed by the factor $m_{\tau} / m_{t}$ and is therefore of the order $10^{-2} M_{\nu_{R}}$. The masses of the neutral $H_{3 r}^{0}$ and $H_{3 i}^{0}$ are even smaller; they are related to the masses of the charged $H_{3}^{ \pm}$and the Standard Model Higgs $H_{2}^{0}$ by equation (73). From the vacuum stability condition $M_{H_{3}^{0}}^{2}>0$ one thus obtains an upper limit on the Standard Model Higgs boson mass $M_{H_{2}^{0}}$ (for a given $M_{\nu_{R}}$ ) or a lower limit on the right-handed mass $M_{\nu_{R}}$ (for a given $M_{H_{2}^{0}}$ ). For example, for $M_{H_{2}^{0}} \approx 60 \mathrm{GeV}$ one finds $M_{\nu_{R}} \gtrsim 5 \mathrm{TeV}$. However, since in the top condensate approach the mass of the Standard Model Higgs is around $2 m_{t}$ (or $\sim m_{t}$ after the renormalization group improvement), one obtains a stronger lower bound on the right handed gauge symmetry breaking scale $M_{\nu_{R}}$ of about $20-50 \mathrm{TeV}$.

The masses of the $\chi_{L}$ scalars [equations $\left.(74),(75)\right]$ vanish in the limit $\left(\lambda_{2}-\lambda_{1}\right) \rightarrow 0$ (i.e. $g_{2} \rightarrow 0$ ) and $m_{\tau} \rightarrow 0$. This fact has a simple interpretation. In the limit $\lambda_{2}=\lambda_{1}$ (which corresponds to the fermion-bubble level) the $\left(\chi_{L}, \chi_{R}\right)$ sector of the effective Higgs potential [equation (56)] depends on $\chi_{L}$ and $\chi_{R}$ only through the combination $\left(\chi_{L}^{\dagger} \chi_{L}+\chi_{R}^{\dagger} \chi_{R}\right)$. This implies that the potential has a global SU(4) symmetry which is larger than the initial $\mathrm{SU}(2)_{L} \times \mathrm{SU}(2)_{R} \times \mathrm{U}(1)_{B-L}$ symmetry. After $\chi_{R}^{0}$ gets a non-vanishing vacuum expectation value $v_{R}$, the symmetry is broken down to $\mathrm{SU}(3)$, resulting in $15-8=7$ Goldstone bosons. Three of them $\left(\chi_{R}^{ \pm}\right.$and $\left.\operatorname{Im} \chi_{R}^{0}\right)$ are eaten by the $\mathrm{SU}(2)_{R}$ gauge bosons $W_{R}^{ \pm}$and $Z_{R}$, and the remaining four $\left(\chi_{L}^{ \pm}, \operatorname{Re} \chi_{L}^{0}\right.$ and $\left.\operatorname{Im} \chi_{R}^{0}\right)$ are physical massless Goldstone bosons. The $\operatorname{SU}(4)$ symmetry is broken by the $\varphi$-dependent terms in the effective potential and by $\operatorname{SU}(2)$ gauge interactions. As a result, $\chi_{L}^{ \pm}, \operatorname{Re} \chi_{L}^{0}$ and $\operatorname{Im} \chi_{L}^{0}$ acquire small masses and become pseudo-Goldstone bosons. In fact, the origin of this approximate SU(4) symmetry can be traced back to the four-fermion operators of equation (48). It is an accidental symmetry resulting from the gauge invariance and parity symmetry of the $G_{7}$ term. Note that no such symmetry occurs in conventional left-right models.

After relaxing the approximation $\kappa^{\prime}=0$, one obtains non-vanishing masses $m_{b}$ and $m_{D}$ (notice that the Yukawa couplings $Y_{2}$ and $Y_{3}$ will also be non-zero in this case). However, these masses are not predicted in the model and can simply be adjusted to desirable values. The Dirac neutrino mass $m_{D}$ is unknown and so remains a free parameter; however, it must be smaller than $m_{\tau}$ in this model in order to satisfy a vacuum stability condition $Y_{4}^{2}-Y_{3}^{2}>0$, which one finds from analysing the potential for the general case [36. This condition is equivalent to $m_{\tau}^{2}-m_{D}^{2}>0$. Also, one finds that the lower bound on $M_{\nu_{R}}$ is strengthened for $\kappa^{\prime} \neq 0$. The Higgs boson masses and mass eigenstates for the general case $\kappa^{\prime} \neq 0$ are only slightly modified and can be also be found in a recent publication [36.

\subsection{Renormalization Group Analysis}

So far the model was analysed in the "bubble approximation", where only fermion and $\mathrm{SU}(2)_{L} \times \mathrm{SU}(2)_{R} \times \mathrm{U}(1)_{B-L}$ gauge boson loops contribute. However, important corrections 
arise from QCD effects and loops with composite Higgs scalars. Those effects can be accounted for [19 by solving the full one-loop renormalization group equations of the low energy effective theory with boundary conditions corresponding to compositeness.

These boundary conditions follow from the vanishing of the radiatively induced kinetic terms for the Higgs scalars at the scale $\Lambda$, where the composite particles break up into their constituents:

$$
Z_{\varphi}\left(\mu^{2} \rightarrow \Lambda^{2}\right)=Z_{\chi}\left(\mu^{2} \rightarrow \Lambda^{2}\right)=Z_{\sigma}\left(\mu^{2} \rightarrow \Lambda^{2}\right)=0
$$

After rescaling the scalar fields to bring their kinetic terms into the canonical form (i.e. normalized to one) one obtains corresponding boundary conditions for the Yukawa and quartic couplings of the low energy effective Lagrangian. These conditions are similar to those obtained by BHL and have the following generic form:

$$
\hat{Y}^{2}=\frac{Y^{2}}{Z} \stackrel{\mu^{2} \rightarrow \Lambda^{2}}{\longrightarrow} \infty, \hat{\lambda}=\frac{\lambda}{Z^{2}} \stackrel{\mu^{2} \rightarrow \Lambda^{2}}{\longrightarrow} \infty, \frac{\hat{\lambda}}{\hat{Y}^{4}}=\frac{\lambda}{Y^{4}} \stackrel{\mu^{2} \rightarrow \Lambda^{2}}{\longrightarrow} 0 .
$$

The renormalized parameters of the model derived in bubble approximation already satisfy the compositeness conditions; for example, the renormalized Yukawa couplings are

$$
\begin{aligned}
& \hat{Y}_{1}^{2}(\mu)=\frac{Y_{1}^{2}}{Z_{\varphi}} \approx\left[\frac{3}{16 \pi^{2}} \ln \left(\frac{\Lambda^{2}}{\mu^{2}}\right)\right]^{-1} ; \quad\left(\hat{Y}_{4} \ll \hat{Y}_{1}\right), \\
& \hat{Y}_{4}^{2}(\mu) \approx \frac{Y_{4}^{2}}{Y_{1}^{2}} \hat{Y}_{1}^{2}(\mu)=\frac{G_{3}}{G_{1}} \hat{Y}_{1}^{2}(\mu), \\
& \hat{Y}_{5}^{2}(\mu)=\frac{Y_{5}^{2}}{Z_{\chi}}=\left[\frac{1}{16 \pi^{2}} \ln \left(\frac{\Lambda^{2}}{\mu^{2}}\right)\right]^{-1}, \\
& \hat{Y}_{6}^{2}(\mu)=\frac{Y_{6}^{2}}{Z_{\sigma}}=\left[\frac{2}{16 \pi^{2}} \ln \left(\frac{\Lambda^{2}}{\mu^{2}}\right)\right]^{-1} .
\end{aligned}
$$

Obviously they diverge as $\mu \rightarrow \Lambda$. Furthermore, their running coincides exactly with that described by the fermion loop contributions (the trace terms) to one-loop $\beta$-functions of the corresponding left-right-symmetric theory. The idea is now to identify the Landau poles in the full one-loop renormalization group evolution of couplings with the compositeness scale $\Lambda$ and run the couplings down to low energy scales.

We will first consider the simplified scenario with $\kappa^{\prime}=0$. In the one-generation scenario the renormalization group equations for the Yukawa couplings in the limit $\kappa^{\prime}=0$ (which requires $Y_{2}=Y_{3}=0$, see section 6.3.1) reduce to ${ }^{12}$

$$
\begin{aligned}
& 16 \pi^{2} \frac{d Y_{1}}{d t}=5 Y_{1}^{3}+Y_{1} Y_{4}^{2}-\left(8 g_{3}^{2}+\frac{9}{2} g_{2}^{2}+\frac{1}{6} g_{1}^{2}\right) Y_{1} \\
& 16 \pi^{2} \frac{d Y_{4}}{d t}=3 Y_{4}^{3}+3 Y_{4} Y_{1}^{2}+Y_{4} Y_{5}^{2}-\left(\frac{9}{2} g_{2}^{2}+\frac{3}{2} g_{1}^{2}\right) Y_{4},
\end{aligned}
$$

\footnotetext{
${ }^{12}$ In the following we will omit the hats over the renormalized quantities.
} 


$$
\begin{aligned}
16 \pi^{2} \frac{d Y_{5}}{d t} & =\frac{7}{2} Y_{5}^{3}+Y_{5}\left(Y_{4}^{2}+\left[2 Y_{6}^{2}\right]\right)-\left(\frac{9}{4} g_{2}^{2}+\frac{3}{4} g_{1}^{2}\right) Y_{5} \\
16 \pi^{2} \frac{d Y_{6}}{d t} & =[6] Y_{6}^{3}+4 Y_{6} Y_{5}^{2} .
\end{aligned}
$$

For large values of $Y_{i}$ the $Y_{i}^{3}$-contributions in the $\beta$-functions are dominant; they quickly drive the couplings down to values of order one as the scale $\mu$ decreases. In this regime gauge and other Yukawa coupling contributions become important, and the interplay of these contributions and $Y_{i}^{3}$ terms result in so-called infrared quasi-fixed points 443. Thus a large range of initial values of the Yukawa couplings at the cutoff is focused into a small range at low energies. The masses of the fermions will then be given implicitly by conditions of the kind $Y(m) \cdot \mathrm{VEV}=m$.

To evolve the Yukawa couplings with one-loop $\beta$-functions to their Landau poles, i.e. in the non-perturbative regime, may appear questionable. However, it has been argued [19, 44] that this should not result in any significant errors. First the running time $t=\ln \mu$ in the non-perturbative domain is only a few percent of the total running time. Second, and more importantly, the infrared quasi-fixed point structure of the renormalization group equations makes the predictions fairly insensitive to the detailed behaviour of the solutions in the large Yukawa coupling domain. Lattice gauge theory has generally confirmed the reliability of perturbation theory in this fixed point analysis 44.

Except for switching to the Standard Model renormalization group equations below the parity breaking scale the only relevant threshold effects in the evolution are due to the masses of the $\sigma$ scalars. From the vacuum structure analysis in bubble approximation, the vacuum expectation values of $\sigma$ and $\chi_{R}$ do not coexist; phenomenology then dictates the choice $\sigma_{0}=0, v_{R} \neq 0$, which requires the four-fermion coupling $G_{8}$ to be sub-critical, or at least satisfying the condition (63). We will assume that the same holds true beyond the bubble approximation and consider $\sigma$ to be non-propagating, or at least decoupled from the low-energy spectrum of the model. Therefore the effects from propagating $\sigma$ scalars can be switched off directly at the cutoff by neglecting the contributions in square brackets of equations (84) and (85). In this limit the running of $Y_{6}$ does not influence the running of the other Yukawa couplings.

In numerical calculations a large number $Y_{i}(\Lambda)$ must be used as boundary condition for Yukawa couplings instead of infinity. Fortunately, the infrared quasi-fixed point structure of the renormalization group equation makes the solutions fairly insensitive to the actual values of $Y_{i}(\Lambda)$ provided that they are large enough [19, 43. In fact, the infrared quasifixed-point behaviour sets in already for $Y_{i}(\Lambda) \gtrsim 5$. The calculations were performed using $Y_{5}(\Lambda)=10$, but taking e.g. $10^{3}$ instead of 10 results only in a correction of about $0.4 \%$ in the low-energy value of $Y_{5}$. The fermion-loop results of equations (78)-(81) imply a fixed ratio between the running coupling constants which one could, as a first approximation, also impose as the boundary condition at the cutoff for the full renormalization group evolution, e.g. $Y_{1}(\Lambda)=3 Y_{5}(\Lambda)=30$. Fortunately, once again, the numerical results depend very weakly on this scaling factor, and for this purpose it could just as well be 
taken to be unity. Figure 4 shows $Y_{5}(\mu)$ obtained by numerically solving equations (82)-

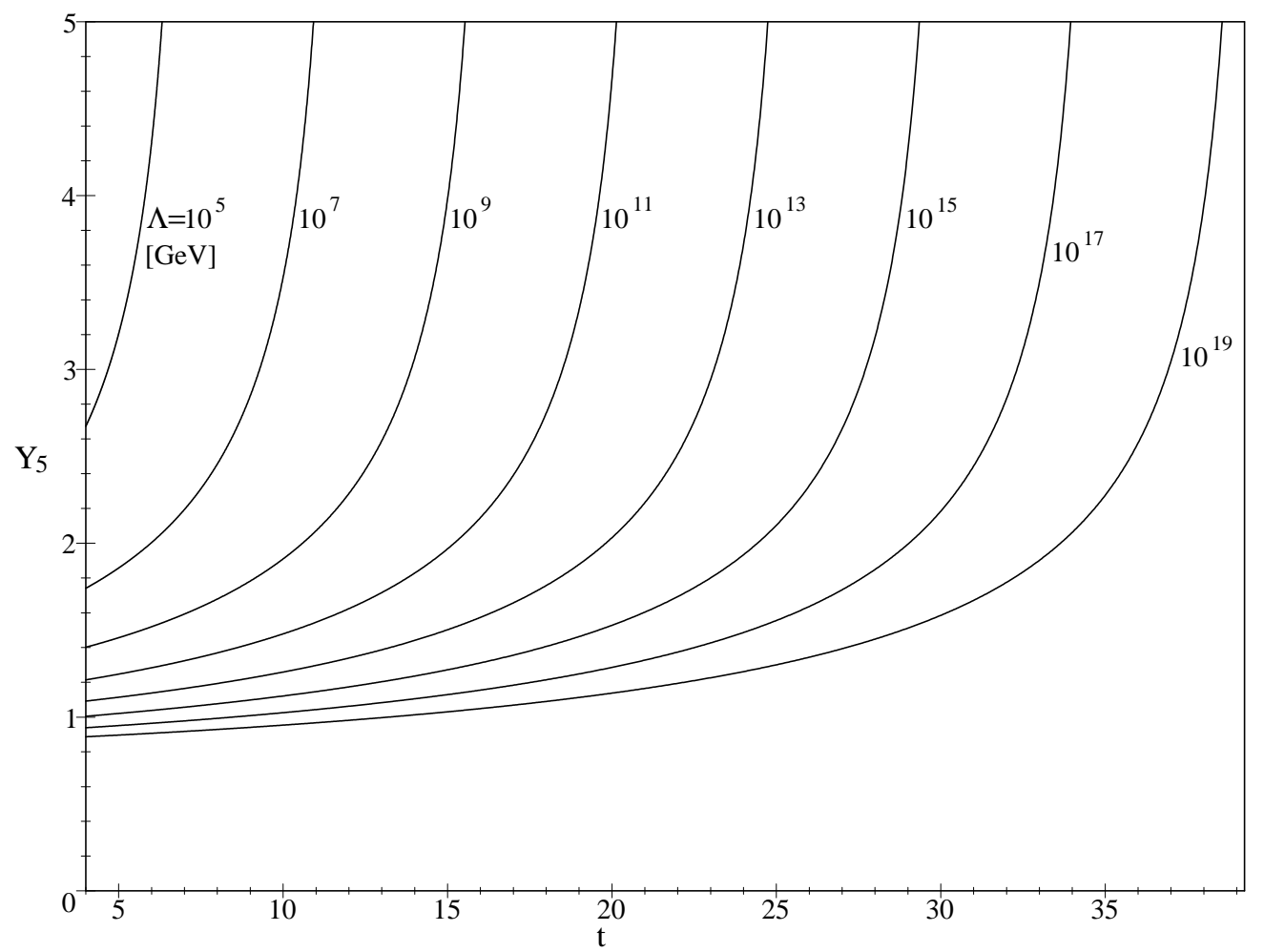

Figure 4: Renormalization group evolution of the $Y_{5}$ Yukawa coupling for various compositeness scales $\Lambda, t=\ln \left(\mu / m_{Z}\right)$.

(84) for various values of the cutoff $\Lambda$. The heavy neutrino mass $M_{\nu_{R}}$ is determined by the equation

$$
M_{\nu_{R}}=Y_{5}\left(M_{\nu_{R}}\right) \cdot \hat{v}_{R}
$$

and for $v_{R} \sim \mu_{R} \ll \Lambda$ one finds values of $Y_{5}\left(M_{\nu_{R}}\right) \simeq Y_{5}\left(\mu_{R}\right)$ roughly between 1 and 2 .

The evolution of $Y_{1}$ and $Y_{4}$ below the parity breaking scale is determined by the usual Standard Model $\beta$-functions [46. It turns out that the numerically most important difference between the left-right and Standard Model $\beta$-functions for $Y_{1}$ is a contribution of $1 / 2 Y_{1}^{3}$ coming from the self energy diagram with a $\varphi_{2}^{+}$scalar exchange. Since the mass of $\varphi_{2}^{+}$is not of the order of the right-handed scale but is suppressed by a factor $\approx 10^{-2}$ we switch to the Standard Model $\beta$-functions two orders of magnitude below the parity breaking scale $\mu_{R}$.

In figure 5 we present the numerical solutions for $Y_{1}(\mu)$ for various values of $\Lambda$ and $\mu_{R}$. One can clearly see the infrared quasi-fixed point structure of the solutions. The values of $Y_{1}$ at $t=0\left(\mu=m_{Z}\right)$ are to some extent sensitive to the magnitude of the cutoff but fairly insensitive to the scale where parity breaks. This is because in fact the 


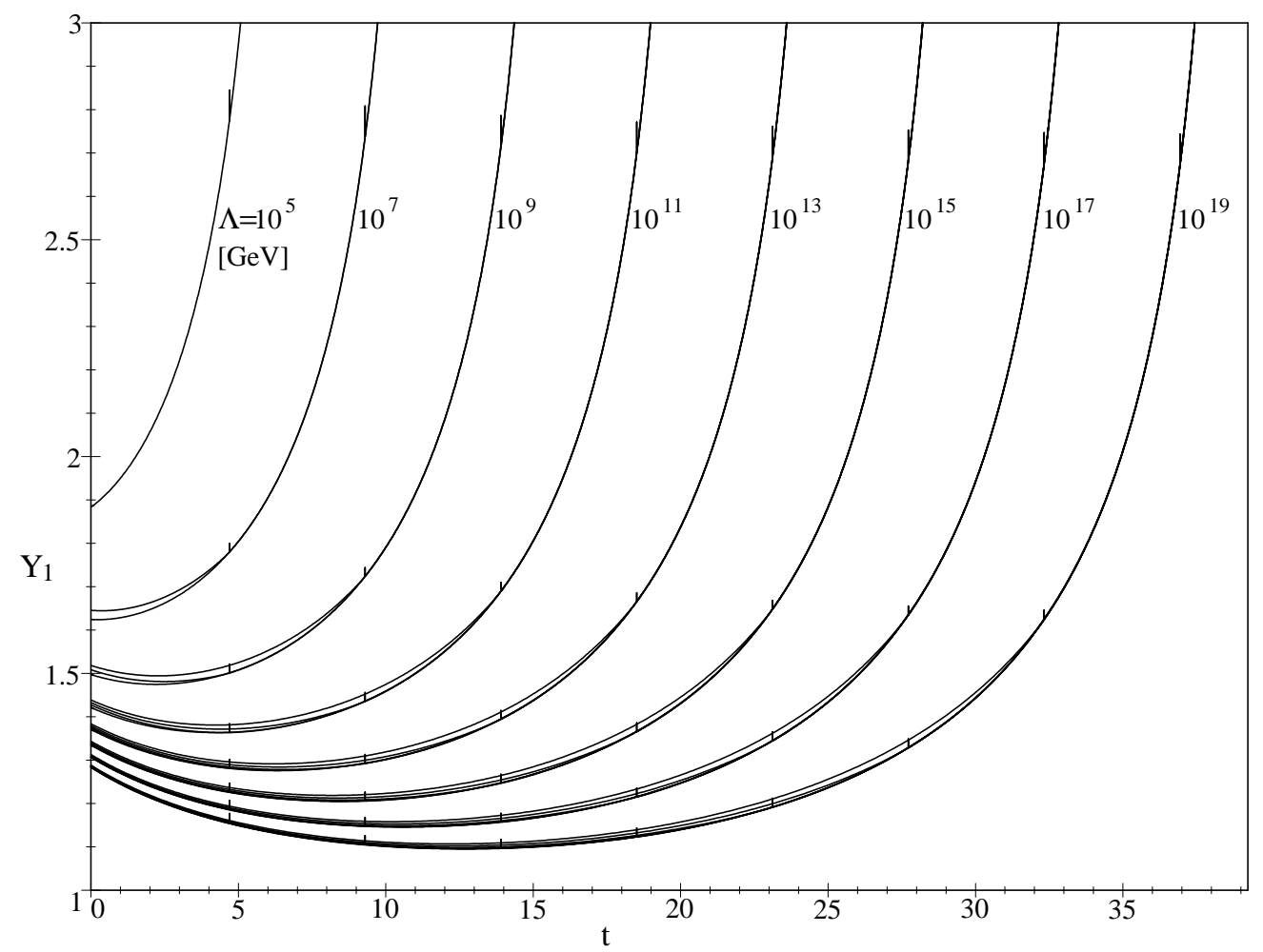

Figure 5: Renormalization Group evolution of the $Y_{1}$ Yukawa coupling for various parity breaking scales $\mu_{R}$ (indicated by little ticks) and compositeness scales $\Lambda ; t=\ln \left(\mu / m_{Z}\right)$.

previously mentioned contribution to the $Y_{1} \beta$-function from the $\varphi_{2}^{+}$exchange makes only a relatively small difference between $(9 / 2) Y_{1}^{3}$ in the Standard Model and $5 Y_{1}^{3}$ in the left-rightsymmetric model with a bi-doublet. For the same reason the top quark mass prediction in the $\kappa^{\prime}=0$ case is very similar to the one of the BHL model [19], which is too high compared to the recent experimental results [41]. Even for a rather high cutoff $\Lambda \approx 10^{17} \mathrm{GeV}$ one obtains a top quark mass about $229 \mathrm{GeV}$.

Because the $\tau$ lepton is much lighter than the top quark, the $\tau$-Yukawa coupling is not governed by any fixed point; instead its low energy value depends essentially on the value at the cutoff. Consequently, the $\tau$ contributes only very little to the composite Higgs bi-doublet, which is driven by large $Y_{1}$ and not by $Y_{4}$. In other words, the model exhibits a top condensate (along with a heavy-neutrino condensate) rather than a tau condensate. One can readily obtain a suitable low-energy value of $Y_{4}$ by choosing a proper value of $Y_{4}(\Lambda)$. Therefore, although $m_{\tau}$ is not predicted in this framework, it can be easily adjusted to the correct value.

So far it was assumed that only one of the two neutral components of the bi-doublet $\varphi$ acquires a vacuum expectation value $\left(\kappa \neq 0, \kappa^{\prime}=0\right)$. Apparently, the resulting fixed-point 
value of the top quark Yukawa coupling is in this limit outside the phenomenologically acceptable region [41]. Moreover, the assumption $\kappa^{\prime}=0$ implies a zero bottom quark mass. Evidently, for this model to be realistic with $m_{b} \neq 0$ one requires either $\kappa^{\prime} \neq 0$ or $Y_{2} \neq 0$. In conventional left-right symmetric models these two conditions are unrelated and can be satisfied separately, but in this model $Y_{2} \neq 0$ automatically means $\kappa^{\prime} \neq 0$ and vice versa. In the following we show the results from analysing the renormalization group evolution of the full set of Yukawa couplings for the general case, which indeed leads to viable top and bottom quark masses for a range of values of $\kappa^{\prime} \sim \kappa$.

As we argued previously, there is a stable vacuum for this model with $\kappa, \kappa^{\prime}$ and $v_{R}$ non-vanishing. In the limit of $\kappa, \kappa^{\prime} \ll v_{R}$ the ratio of vacuum expectation values is given by

$$
\frac{\kappa^{\prime}}{\kappa}=-\frac{Y_{3}}{Y_{4}}+\mathcal{O}\left(\frac{\kappa^{2}}{v_{R}^{2}}\right) .
$$

Without loss of generality we assume $\left|\kappa^{\prime}\right|<|\kappa|$. On the other hand, to obtain $m_{t} \gg m_{b}$ for $\kappa \sim \kappa^{\prime}$ one requires the condition

$$
\frac{Y_{2}}{Y_{1}} \approx-\frac{\kappa^{\prime}}{\kappa}
$$

to be satisfied. Since the lepton Yukawa couplings in equation (87) are not governed by infrared quasi-fixed points and depend on the corresponding boundary conditions at the cutoff, the ratio of vacuum expectation values

$$
\tan \beta \equiv \frac{\kappa}{\kappa^{\prime}}
$$

is essentially a free parameter of the model. The full one-loop renormalization group equations for the Yukawa sector are found to be

$$
\begin{aligned}
& 16 \pi^{2} \frac{d Y_{1}}{d t}= 5 Y_{1}^{3}+7 Y_{1} Y_{2}^{2}+Y_{1}\left(Y_{3}^{2}+Y_{4}^{2}\right) \\
&+2 Y_{2} Y_{3} Y_{4}-\left(8 g_{3}^{2}+\frac{9}{2} g_{2}^{2}+\frac{1}{6} g_{1}^{2}\right) Y_{1}, \\
& 16 \pi^{2} \frac{d Y_{2}}{d t}= 5 Y_{2}^{3}+7 Y_{2} Y_{1}^{2}+Y_{2}\left(Y_{3}^{2}+Y_{4}^{2}\right) \\
&+2 Y_{1} Y_{3} Y_{4}-\left(8 g_{3}^{2}+\frac{9}{2} g_{2}^{2}+\frac{1}{6} g_{1}^{2}\right) Y_{2}, \\
& 16 \pi^{2} \frac{d Y_{3}}{d t}=3 Y_{3}^{3}+Y_{3}\left(Y_{4}^{2}+Y_{5}^{2}\right)+3 Y_{3}\left(Y_{1}^{2}+Y_{2}^{2}\right) \\
&+6 Y_{1} Y_{2} Y_{4}-\left(\frac{9}{2} g_{2}^{2}+\frac{3}{2} g_{1}^{2}\right) Y_{3}, \\
& 16 \pi^{2} \frac{d Y_{4}}{d t}=3 Y_{4}^{3}+Y_{4}\left(Y_{3}^{2}+Y_{5}^{2}\right)+3 Y_{4}\left(Y_{1}^{2}+Y_{2}^{2}\right)
\end{aligned}
$$




$$
\begin{array}{r}
+6 Y_{1} Y_{2} Y_{3}-\left(\frac{9}{2} g_{2}^{2}+\frac{3}{2} g_{1}^{2}\right) Y_{4} \\
16 \pi^{2} \frac{d Y_{5}}{d t}=\frac{7}{2} Y_{5}^{3}+Y_{5}\left(Y_{3}^{2}+Y_{4}^{2}\right)-\left(\frac{9}{4} g_{2}^{2}+\frac{3}{4} g_{1}^{2}\right) Y_{5} .
\end{array}
$$

For a heavy, non-propagating $\sigma$-scalar the coupling $Y_{6}$ decouples. Evolving this set of Yukawa couplings down to low energies one finds that now the square root $\sqrt{Y_{1}^{2}+Y_{2}^{2}}$ exhibits a fixed point behaviour (just like $Y_{1}$ for the case $Y_{2}=0$ ), whereas the ratio of $Y_{1}$ and $Y_{2}$ runs very slowly (see figure 6). Thus the ratio $Y_{2} / Y_{1}$ at low energies depends in a straightforward way on the boundary condition at the cutoff. Unfortunately, the

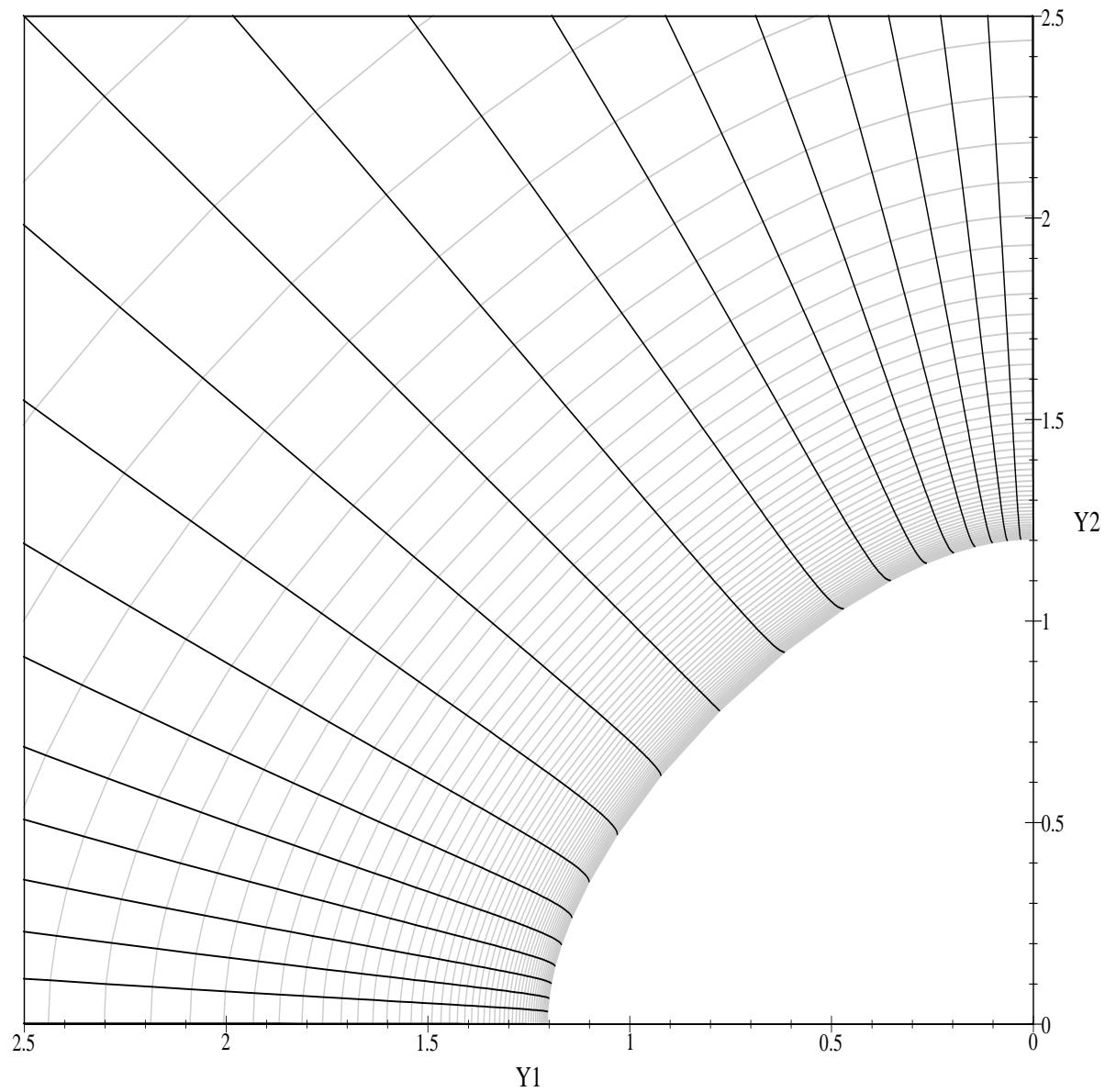

Figure 6: Location of fixed points in the $Y_{1}-Y_{2}$-plane for $\Lambda=10^{15} \mathrm{GeV}$ and $\mu_{R}=10^{6} \mathrm{GeV}$.

renormalization group evolution of Yukawa couplings does not automatically impose the relation (88), since the boundary conditions on lepton and quark Yukawa couplings are unrelated when starting from the four-fermion Lagrangian. Thus, at this level one does not naturally explain the large top-bottom mass splitting. However, since the ratios of Yukawa couplings run only slowly from high to low energies, at least there exist sensible boundary 
conditions that lead, with (87), to the desired situation (88) at low energies, which itself is a non-trivial point. Therefore one may assume that the relation (88) is satisfied and leave it to the underlying physics to justify this choice.

Below the right-handed scale one should switch to the Standard Model $\beta$-functions of $Y_{t}$ and $Y_{b}$, which are obvious linear combinations of $Y_{1}$ and $Y_{2}$. Imposing the relation (88) as argued above, one can find those values of $\tan \beta$ which lead to the correct top mass, depending on the values of the cutoff $\Lambda$ and the right-handed scale $\mu_{R}$.

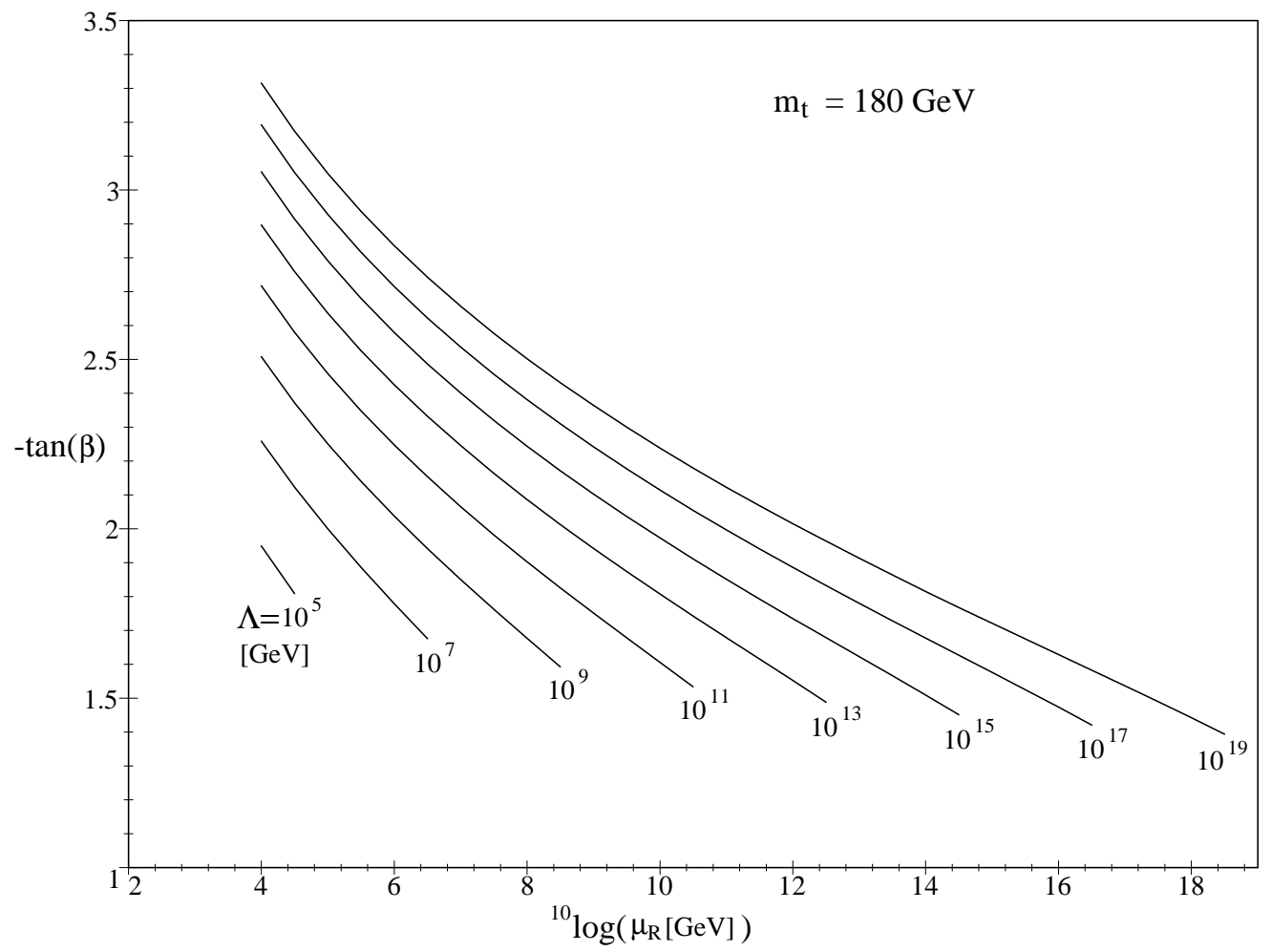

Figure 7: Values of $\tan \beta$ for $m_{t}=180 \mathrm{GeV}$ and various magnitudes of the cutoff $\Lambda$ and righthanded scale $\mu_{R}$.

Figure 7 shows the results for $\tan \beta$ assuming a top mass of $m_{t}=180 \mathrm{GeV}$. One observes that a viable top mass can be obtained for a large range of possible values of the cutoff and for various parity breaking scales. This is in contrast with the top condensate approach to the Standard Model [19] where the lowest possible (but still too high) top mass arises for the largest possible cutoff. As one can see from the figure, this model can reproduce a viable top quark mass for values of the cutoff as low as $200 \mathrm{TeV}$ and for a parity breaking scale about $50 \mathrm{TeV}$. This means that there is only a minimal amount of fine-tuning involved and the gauge hierarchy problem gets significantly ameliorated. If one considers different values for the top mass, the whole set of curves in figure 0 is slightly 
shifted vertically, e.g., for $m_{t}=(168-192) \mathrm{GeV}$ one finds an overall range for $\tan \beta$ of $(1.3-4.0)$. Further phenomenological studies [35, 36] showed that this model is compatible with all experimental data.

Since the model was constructed in order to achieve a Standard Model decoupling limit, the phenomenological viability seems not too surprising. However, it is nontrivial to obtain a scenario where the symmetry breaking pattern works in the desired way and the top and Higgs mass predictions are not in contradiction with the data. The model discussed above has altogether 9 input parameters (eight four-fermion couplings $G_{1}, \ldots G_{8}$ and the scale of new physics $\Lambda$ ), a factor of two less than the number of free parameters in conventional left-right-symmetric models. It is thus constrained compared to the conventional model, and it was demonstrated that, e.g., for Higgs scalars in the triplet representation the correct pattern of symmetry breaking does not arise. We have shown that with scalar doublets the model allows the required features; in particular, parity breaking at low energies occurs automatically regardless of the choice of the parameters of the model, provided the gauge symmetry breaks at all. This is in striking contrast with conventional left-right models, in which for parity to be spontaneously broken the parameters of the Higgs potential must be chosen so as to satisfy a certain inequality. At the same time, the number of parameters to be tuned in order to achieve the correct hierarchy of the symmetry breaking scales is the same as in the conventional approach; in this respect the composite model has no advantages over conventional left-right models.

\section{Summary}

We emphasized that the Standard Model describes the existing data with remarkable accuracy. The success of the Standard Model implies that any theory of new physics will be phenomenologically viable if it has a suitable Standard Model limit. By Standard Model limit we mean models which become, for certain parameter choices, indistinguishable from the Standard Model, and where the masses and couplings lie in the range allowed by the data. At the moment the Standard Model limit simply allows a scenario to hide behind the Standard Model, but it also ensures that potential deviations in the future can be understood as corrections to this limit.

Obviously the Standard Model limit implies that no extra light particles exist which should have already been detected. There are further direct restrictions for extra couplings and masses which stem, for example, from rare decays and FCNC limits. Additional indirect limitations arise from radiative corrections. The agreement between the top mass value derived from radiative corrections with its experimental value leaves hardly any room for further custodial $\mathrm{SU}(2)$ violating effects in the electro-weak precision variable $T$. This most likely requires that the electro-weak symmetry breaking operator is a doublet under $\mathrm{SU}(2)_{L}$ with hypercharge $Y=1$. Due to non-decoupling effects this also disfavours models where extra fermionic doublets contribute to $T$ via loops. On the other hand, the "size" of the symmetry breaking sector is limited by the electro-weak precision variable $S$, which 
roughly counts the number of degrees of freedom. The smallness of $S$ probably points already towards the existence of a scalar Higgs particle as unitarity partner of the Goldstone bosons, since typically the contributions of vector like states (which mix and lead to $1 / M^{2}$ corrections) are considerably larger than those of scalars, which only lead to logarithmic contributions. But even if there is a hint for a scalar Higgs particle, this does not yet tell us whether this scalar is composite or fundamental. Therefore, the current data contains no evidence about the nature of the solution to the hierarchy problem and the related new physics beyond the Standard Model at TeV scales. While the MSSM can be viewed as a theory with a Standard Model limit, the difficulties with naive Technicolor are associated with the absence of such a limit. Some modern Technicolor approaches, such as Topcolor assisted Technicolor, essentially work in the direction of reestablishing such a Standard Model limit.

Next we pointed out that it is possible to build models of dynamical symmetry breaking which are systematically more viable due to a Standard Model limit. We evaluated phenomenological guidelines which lead to such scenarios. The most interesting aspect is that dynamics quite different from QCD is required in order to produce a scalar resonance instead of a rho-like vector. An example for a model which has a Standard Model limit is the so-called BHL model of electro-weak symmetry breaking. The BHL model is however unacceptable, since it cannot accommodate the correct top mass. With the help of the Pagels-Stokar relation we argued that this has systematic reasons and that the experimental top mass value is in general too small for a scenario with just a single top condensate. We postulated therefore a sequential breaking of an extended gauge group with a second condensate and more complex relations between the vacuum expectation values and masses.

We presented a left-right-symmetric model which realizes the ideas given above. This model is to our knowledge the first successful attempt to break left-right symmetry dynamically. We find a tumbling scenario where the breaking of parity and $\mathrm{SU}(2)_{R}$ eventually drives the breaking of the electro-weak symmetry. The model gives a viable top quark mass value and exhibits a number of low and intermediate scale Higgs bosons. Furthermore it predicts relations between masses of various scalars and between fermion and Higgs boson masses which are in principle testable. If the right-handed scale $\mu_{R}$ is of the order of a few tens of $\mathrm{TeV}$, the neutral CP-even and CP-odd scalars $\varphi_{2 r}^{0}$ and $\varphi_{2 i}^{0}$ can be even lighter than the electro-weak Higgs boson. In fact, they can be as light as $\sim 50-100 \mathrm{GeV}$ and thus might still be observable at LEP II. To summarize, this model can be made consistent with all experimental data and demonstrates that viable models of electro-weak symmetry breaking can be built. It should be interesting to investigate whether other models can be constructed along these lines.

\section{Acknowledgments}

We would like to thank D. Kominis, J. Manus and G. Triantaphyllou for interesting and useful discussions. This work has been supported in part by DFG grant Li 519/2-2 and in 
part by the Director, Office of Energy Research, Office of High Energy and Nuclear Physics, Division of High Energy Physics of the U.S. Department of Energy under Contract DEAC03-76SF00098. E.S. is supported by a BASF research fellowship and the Studienstiftung des deutschen Volkes.

\section{References}

[1] G. Altarelli, CERN-TH-96-265, Oct 1996, talk given at Cracow International Symposium on Radiative Corrections (CRAD 96), Cracow, Poland, Aug 1996, hepph/9611239.

[2] See e.g. M. Veltman, Acta Phys. Pol. B12 (1981) 437.

[3] See for example T. Appelquist, J. Terning and L.R.C. Wijewardhan, Phys. Rev. Lett. 79 (1997) 2767.

[4] H. P. Nilles, Phys. Rep 110 (1984) 1.

[5] See e.g. M. Dine, A. E. Nelson, Y. Nir and Y. Shirman, Phys. Rev. D53 (1996) 2658; H. Murayama, Phys. Rev. Lett. 79 (1997) 18.

[6] N. Seiberg, Phys. Rev. D49 (1994) 6857; Nucl. Phys. B435 (1995) 129;

For a review see e.g. K. Intriligator and N. Seiberg, Nucl. Phys. Proc. Suppl. 45BC (1996) 1.

[7] H.E. Haber, talk given at the 5th International Conference on Supersymmetries in Physics (SUSY 97), Philadelphia, May 1997, hep-ph/9709450.

[8] H.E. Haber, in Perspectives for Electroweak Interactions in $e^{+} e^{-}$Collisions, Proceedings of the Ringberg Workshop, Ringberg Castle, Tegernsee, 5-8 February, 1995, edited by B.A. Kniehl (World Scientific, Singapore, 1995) pp. 219-231

[9] H.E. Haber, R. Hempfling and A.H. Hoang, Z. Phys. C75 (1997) 539;

M. Carena, J.R. Espinosa, M. Quiros and C.E.M. Wagner, Phys. Lett. B355 (1995) 209;

M. Carena, M. Quiros and C.E.M. Wagner, Nucl. Phys. B461 (1996) 407 and references therein.

[10] M.E. Peskin and T. Takeuchi, Phys. Rev. Lett. 65 (1990) 964, Phys. Rev. D 46 (1992) 381.

[11] See e.g. K. Lane, Proceedings of the International Conference on High Eenergy Physics, Warsaw, Poland, 1996, Eds. Z. Ajduk and A.K. Wroblewski, World Scientific, 1997;

R.S. Chivukula, talk presented at Advanced School on Electroweak Theory, Mao, Menorca, June 1996, hep-ph/9701322; 
K. Lane, An Introduction to Technicolor, Proceedings of the 1993 TASI School, Eds. S. Raby and T. Walker, World Scientific, 1994.

[12] S. Weinberg, Phys. Rev. Lett. 18 (1967) 507.

[13] G. 't Hooft, Nucl. Phys. B72 (1974) 461.

[14] P. Langacker and J. Erler, hep-ph/9703428 and Review of Particle Properties, Phys. Rev. D 54 (1996) 1.

[15] See e.g. W. Hollik, Heavy Flavours II, ed. by A.J. Buras and M. Lindner, World Scientific, Singapore, 1998.

[16] A. Blumhofer and M. Lindner, Nucl. Phys. B407 (1993) 173.

[17] S. Dimopoulos and L. Susskind, Nucl. Phys. B155 (1979) 237;

E. Eichten and K. Lane, Phys. Lett. B90 (1980) 125.

[18] T. Appelquist, T. Takeuchi. M.B. Einhorn and L.C.R. Wijewardhana, Phys. Lett. B232 (1989) 211.

[19] W.A. Bardeen, C.T. Hill and M. Lindner, Phys. Rev. D41 (1990) 1647.

[20] Y. Nambu, in New Theories in Physics, Proc. XI Int. Symposium on Elementary Particle Physics, eds. Z. Ajduk, S. Pokorski and A. Trautman (World Scientific, Singapore, 1989) and EFI report No. 89-08 (1989), unpublished.

[21] A. Miransky, M. Tanabashi and K. Yamawaki, Mod. Phys. Lett. A4 (1989) 1043; Phys. Lett. B221 (1989) 177.

[22] W.J. Marciano, Phys. Rev. Lett. 62 (1989) 2793.

[23] V.G. Vaks and A.I. Larkin, Sov. Phys. JETP 13 (1961) 192.

[24] Y. Nambu and G. Jona-Lasinio, Phys. Rev. 122 (1961) 345.

[25] J.M. Cornwall, R. Jackiw and E. Tomboulis, Phys. Rev. D10 (1974) 2445.

[26] A. Blumhofer, Phys.Lett. B320 (1994) 352; Nucl.Phys. B437 (1995) 25.

[27] C.T. Hill, Phys. Rev. D24 (1981) 691;

C.T. Hill, C.N. Leung and S. Rao, Nucl. Phys. B262 (1985) 517;

J. Bagger, S. Dimopoulos and M. Masso, Phys. Lett. 156B (1985) 357.

[28] C.T. Hill, Phys. Lett. B266 (1991) 419;

For a review see C.T. Hill, invited talk at International Workshop on Perspectives of Strong Coupling Gauge Theories (SCGT 96), Nagoya, Japan, 13-16 Nov 1996, hep$\mathrm{ph} / 9702320$. 
[29] S.P. Martin, Phys. Rev. D45 (1992) 4283.

[30] M. Lindner and D. Ross, Nucl. Phys. B370 (1992) 30.

[31] R. Bönisch, Phys. Lett. B268 (1991) 394.

[32] H. Pagels and S. Stokar, Phys. Rev. D20 (1979) 2947;

A. Carter and H. Pagels, Phys. Rev. Lett. 43 (1979) 1845;

R. Jackiw and K. Johnson, Phys. Rev. D8 (1973) 2386.

[33] J.C. Pati and A. Salam, Phys. Rev. D10 (1975) 275;

R.N. Mohapatra and J.C. Pati, Phys. Rev. D11 (1975) 566; 2558;

G. Senjanović and R.N. Mohapatra, Phys. Rev. D12 (1975) 1502.

[34] R.N. Mohapatra and G. Senjanović, Phys. Rev. Lett. 44 (1980) 912; Phys. Rev. D23 (1981) 165.

[35] E. Akhmedov, M. Lindner, E. Schnapka and J. Valle, Phys. Lett. B368 (1996) 270.

[36] E. Akhmedov, M. Lindner, E. Schnapka and J. Valle, Phys. Rev. D53 (1996) 2752.

[37] T. Eguchi, Phys. Rev. D14 (1976) 2755;

F. Cooper, G. Guralnik and N. Snyderman, Phys. Rev. Lett. 40 (1978) 1620.

[38] G. Senjanović and R.N. Mohapatra, ref. [33]

[39] D. Wyler and L. Wolfenstein, Nucl. Phys. B218 (1983) 205.

[40] See e.g. G. Senjanović, Nucl. Phys. B153 (1979) 334;

C.S. Lim and T. Inami, Prog. Theor. Phys. 67 (1982) 1569;

F.I. Olness and M.E. Ebel, Phys. Rev. D32 (1985) 1769;

J.F. Gunion, J. Grifols, A. Mendez, B. Kayser and F. Olness, Phys. Rev. D40 (1989) 1546 .

[41] P.L. Tipton, Proceedings of the 28th International Conference of High Energy Physics, Warsaw 1996, Eds. Z. Ajduk and A.K. Wroblewski, World Scientific.

[42] N.G. Deshpande, J.F. Gunion, B. Kayser and F. Olness, Phys. Rev. D44 (1991) 837.

[43] C.T. Hill, Phys. Rev. D24 (1981) 691.

[44] A. Blumhofer, R. Dawid and J. Manus, hep-ph/9710495.

[45] See e.g. B. Lin, I. Montvay, G. Münster, M. Plagge and H. Wittig, Phys. Lett. B317 (1993) 143.

[46] See e.g. T.P. Cheng, E. Eichten and L.F. Li, Phys. Rev. D9 (1974) 2259. 

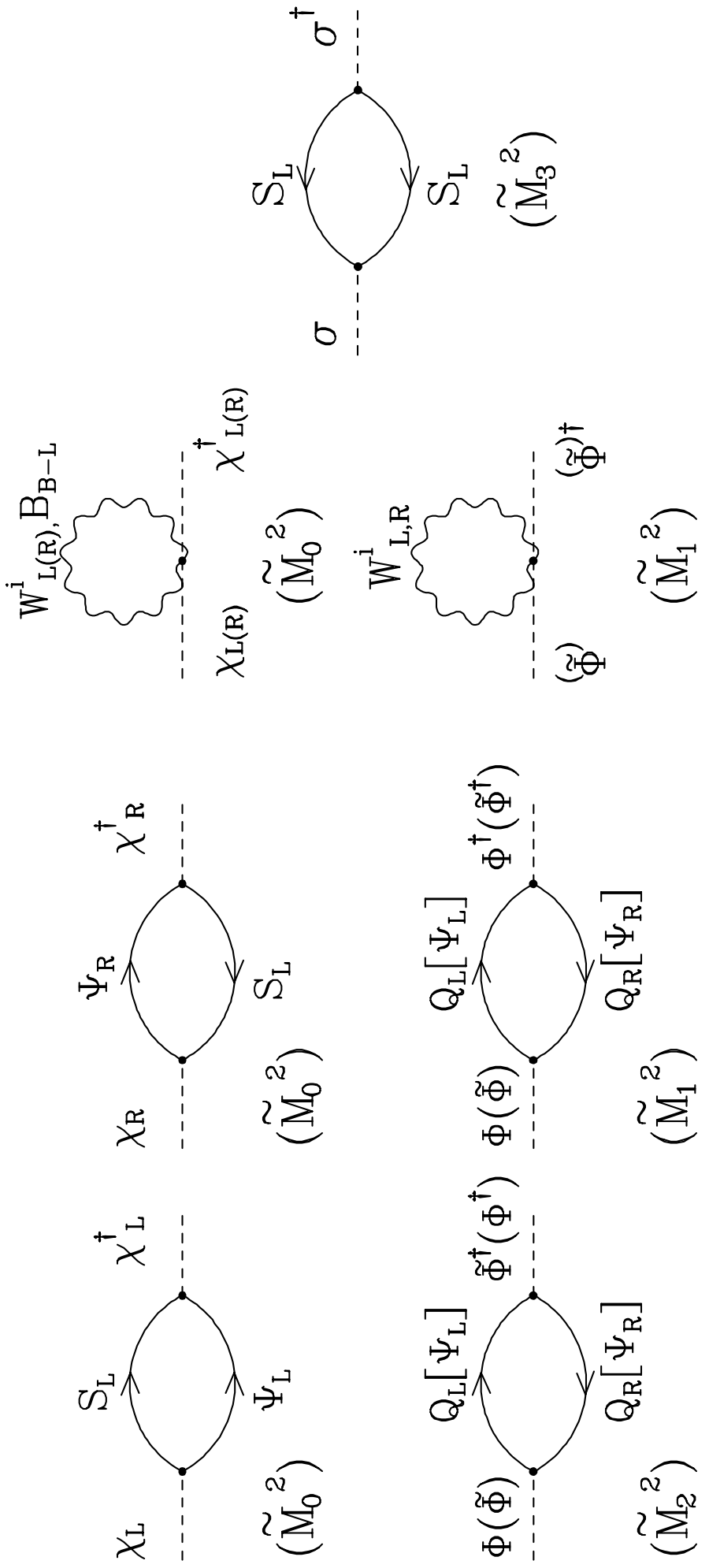

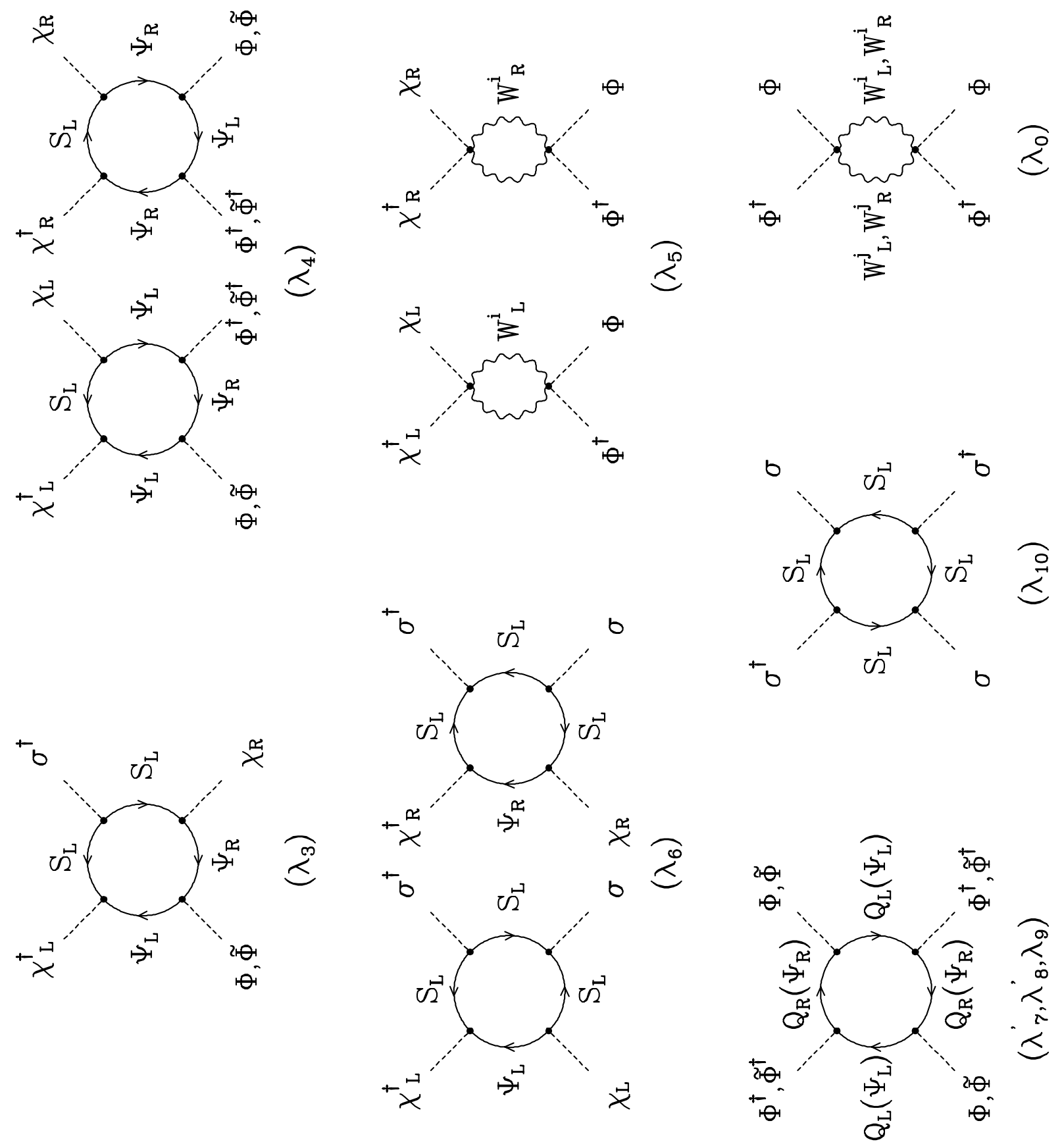


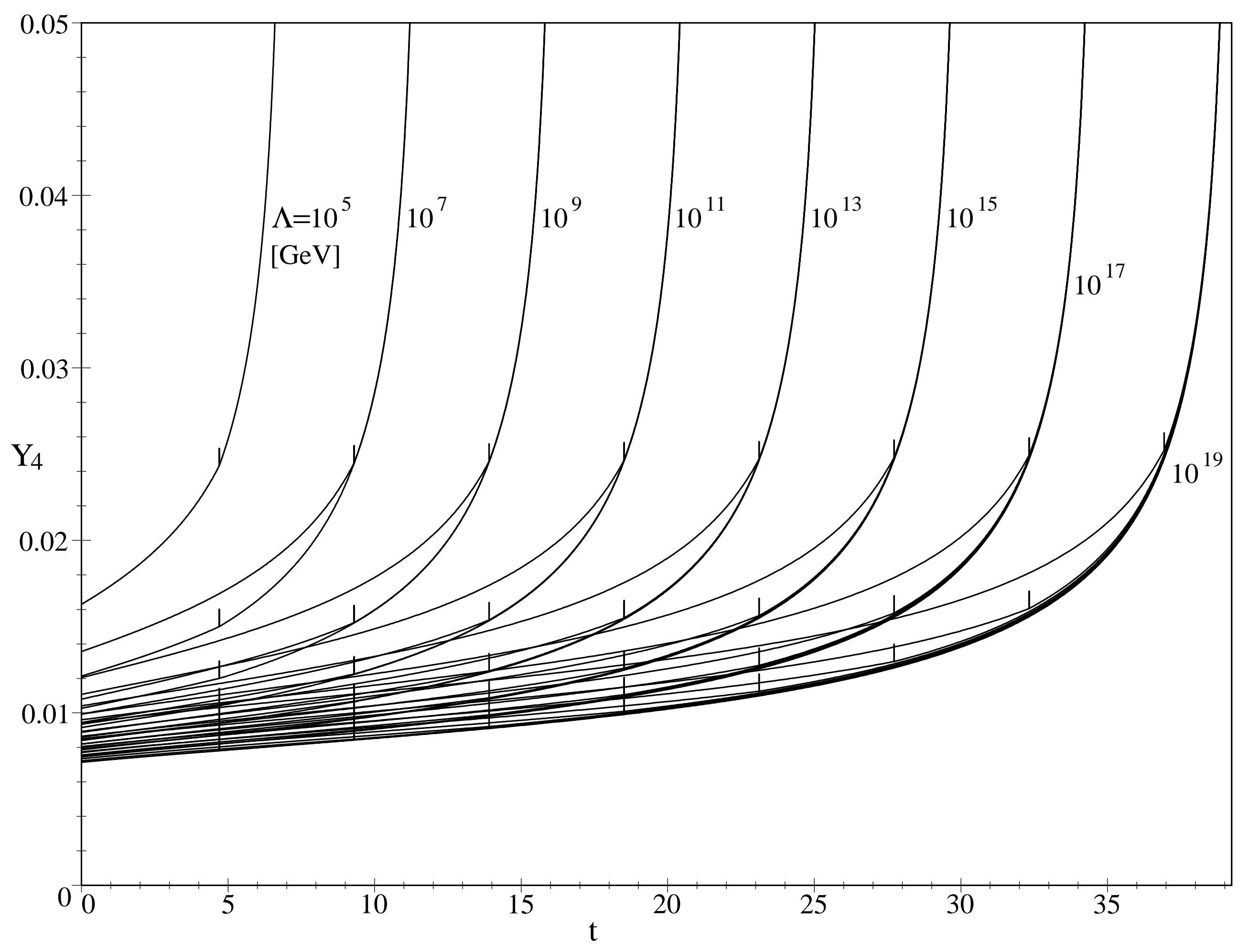

